\title{
New giant radio sources and underluminous radio halos in two galaxy clusters
}

\author{
V. Cuciti ${ }^{1,2}$, G. Brunetti ${ }^{2}$, R. van Weeren ${ }^{3}$, A. Bonafede ${ }^{2}$, D. Dallacasa ${ }^{1,2}$, R. Cassano ${ }^{2}$, T. Venturi $^{2}$, and R. Kale ${ }^{4}$ \\ 1 Dipartimento di Fisica e Astronomia, Università di Bologna, via P. Gobetti 93/2, 40129 Bologna, Italy \\ e-mail: vcuciti@ira.inaf.it \\ 2 INAF-Istituto di Radioastronomia, via P. Gobetti 101, 40129 Bologna, Italy \\ 3 Harvard-Smithsonian Center for Astrophysics, 60 Garden Street, Cambridge, MA 02138, USA \\ 4 National Centre for Radio Astrophysics, Tata Institute of Fundamental Research, Post Bag 3, 411007 Pune, India
}

Received 15 May 2017 / Accepted 16 September 2017

\section{ABSTRACT}

\begin{abstract}
Aims. The aim of this work is to analyse the radio properties of the massive and dynamically disturbed clusters Abell 1451 and Zwcl $0634.1+4750$, especially focusing on the possible presence of diffuse emission.

Methods. We present new Giant Metrewave Radio Telescope (GMRT) 320 MHz and Jansky Very Large Array (JVLA) 1.5 GHz observations of these two clusters.

Results. We found that both Abell 1451 and Zwcl $0634.1+4750$ host a radio halo with a typical spectrum $(\alpha \sim 1-1.3)$. Similarly to a few other cases reported in the recent literature, these radio halos are significantly fainter in radio luminosity with respect to the current radio power-mass correlations and they are smaller than classical giant radio halos. These underluminous sources might contribute to elucidating the complex mechanisms of formation and evolution of radio halos. Furthermore, we detected a candidate radio relic at large distance from the cluster centre in Abell 1451 and a peculiar head-tail (HT) radio galaxy in Zwcl 0634.1+4750, which might be interacting with a shock front.
\end{abstract}

Key words. galaxies: clusters: individual: Abell 1451, Zwcl 0634.1+4750 - galaxies: clusters: intracluster medium radiation mechanisms: non-thermal

\section{Introduction}

Distorted radio galaxies with complex morphologies are often observed in galaxy clusters (e.g. Willson 1970; Hill \& Longair 1971; Wellington et al. 1973; Eilek et al. 1984; O’Dea \& Owen 1986; Pinkney et al. 1994; Feretti et al. 2001; Feretti \& Venturi 2002; Lane et al. 2002; Giacintucci \& Venturi 2009; Mao et al. 2009; Owen et al. 2014). These spectacular sources are the result of the interaction between the radio galaxies and the dense surrounding intra cluster medium (ICM), which causes the bending of the jets and lobes of the radio galaxies (Jaffe \& Perola 1973; Begelman et al. 1979; Owen \& Rudnick 1976; Miley 1980). In addition, some galaxy clusters host diffuse synchrotron emission associated with relativistic particles and magnetic fields in the ICM. Depending on their location and morphology, these cluster-scale radio sources are referred to as radio halos or relics. Radio halos are located at the centre of clusters and their morphology roughly follows the X-ray emission from the thermal ICM. Radio relics are situated at the periphery of the clusters and they typically have elongated or arc-like shapes (Feretti et al. 2012). There is compelling evidence that radio halos and relics are hosted by dynamically disturbed systems, while clusters with no cluster-scale radio emission are generally relaxed (Cassano et al. 2010, 2012, 2013; Cuciti et al. 2015; de Gasperin et al. 2014). These sources suggest that part of the gravitational energy injected in the form of shocks and large scale gas motions is drained into non-thermal components through a hierarchy of complex mechanisms (Brunetti \& Jones 2014).
In the current theoretical picture, radio halos form via the acceleration of relativistic electrons by means of clusterwide turbulence injected in the ICM during merging events (Brunetti et al. 2001; Petrosian 2001). Alternatively, it has been proposed that radio halos are caused by secondary electrons generated by proton-proton collisions (Dennison 1980; Blasi \& Colafrancesco 1999), however, the non-detection of galaxy clusters in the $\gamma$-rays disfavours this possibility (Ackermann et al. 2010, 2014, 2016; Jeltema \& Profumo 2011; Brunetti et al. 2012, 2017; Zandanel \& Ando 2014). Models based on the combination of the two mechanisms, where secondary electrons are reaccelerated by turbulence, have also been proposed (Brunetti \& Blasi 2005; Brunetti \& Lazarian 2011; Pinzke et al. 2017; Brunetti et al. 2017).

There is broad consensus that radio relics trace shock fronts propagating through the ICM (Roettiger et al. 1999; Ensslin et al. 1998; Pinzke et al. 2013; Brüggen et al. 2012; Brunetti \& Jones 2014, for reviews). However, the commonly used diffusive shock acceleration (DSA) model is challenged by the low Mach number of shocks associated with cluster mergers $(\mathcal{M} \sim 1-3)$. Therefore, the re-acceleration of a population of relativistic electrons might be required to explain the radio brightness of some relics (Markevitch et al. 2005; Kang et al. 2012; Vazza \& Brüggen 2014; Bonafede et al. 2014; Shimwell et al. 2015; Eckert et al. 2016; Botteon et al. 2016; van Weeren et al. 2016, 2017).

There is a correlation between the radio power of radio halos and the X-ray luminosity of the hosting clusters, with more powerful radio halos being found in more luminous 
Table 1. Clusters properties.

\begin{tabular}{lccccc}
\hline \hline Name & $\begin{array}{c}\text { RA and Dec } \\
(J 2000)\end{array}$ & $z^{*}$ & $\begin{array}{c}D_{L} \\
(\mathrm{Mpc})\end{array}$ & $\begin{array}{c}M_{500}^{*} \\
\left(10^{14} M_{\odot}\right)\end{array}$ & $\begin{array}{c}L_{500}^{* *}[0.1-2.4] \mathrm{kev} \\
\left(10^{44} L_{\odot}\right)\end{array}$ \\
\hline Abell 1451 & $120316.2-213212.7$ & 0.199 & 978.6 & 7.32 & 6.61 \\
ZwCl 0634.1+4750 & $063802.5+474723.8$ & 0.174 & 839.6 & 6.52 & 4.72 \\
\hline
\end{tabular}

References. ${ }^{(*)}$ Planck Collaboration XXIX (2014), ${ }^{(* *)}$ Piffaretti et al. (2011).

clusters (Liang et al. 2000; Bacchi et al. 2003; Cassano et al. 2006; Rudnick \& Lemmerman 2009). Also, galaxy clusters show a bimodal behaviour in the radio power-X-ray luminosity diagram: radio halos follow the correlation, while upper limits to the radio power of clusters without diffuse emission are well below this scaling relation (Brunetti et al. 2007). A similar correlation has been found between the radio power of radio halos and the integrated Sunyaev-Zel'dovich (SZ) effect signal (Basu 2012; Cassano et al. 2013; Martinez Aviles et al. 2016) as well as the bimodality of clusters in the radio power-SZ diagram connected with the dynamical status of the hosting clusters (Cassano et al. 2013). Both the X-ray luminosity and the SZ signal are used as proxies of the mass of the clusters, but the SZ signal should be less affected by the dynamical state of the clusters and it is considered to be a more robust indicator of the cluster mass than the X-ray luminosity (Motl et al. 2005; Nagai 2006).

A correlation between the radio power of radio relics and the mass of the host cluster has recently been found, together with a trend of larger relics to be located at larger distances from the cluster centre (de Gasperin et al. 2014).

Specifically, for radio halos, the radio bimodality of galaxy clusters and the distribution of clusters in the radio-mass or radio- $L x$ diagram can be explained in terms of superposition of different evolutionary stages of the cluster life, in connection with their dynamical activity (e.g. Brunetti et al. 2009; Cassano et al. 2016). In this respect, idealised simulations of a -single- two-body merger by Donnert et al. (2013), which include turbulent re-acceleration of primary electrons, are very instructive. They show that merger-induced turbulence has the potential to light up the radio emission, lifting the clusters from the region of the upper limits in the $P_{1.4 \mathrm{GHz}}-L_{\mathrm{X}}$ diagram up to the region spanned by the $P_{1.4 \mathrm{GHz}}-L_{\mathrm{X}}$ correlation. Within $\sim 1-$ $2 \mathrm{Gyr}$, the radio luminosity is expected to decrease and the clusters go back to the upper limit region. According to these simulations, clusters are expected to spend $\leq 0.5 \mathrm{Gyr}$ in the region between the correlation and the upper limits, thus this region would be statistically less populated (Donnert et al. 2013). A complication comes from the possibility that secondary particles injected by hadronic collisions may contribute to the cluster-scale radio emission. The level of this contribution is still not understood; it should become dominant in the cases where turbulent acceleration is not efficient enough. This leads to the expectation of "off state" halos, produced in less turbulent systems, where the synchrotron emission is maintained by the continuous injection of secondary particles generated from proton-proton collisions (e.g. Brunetti \& Lazarian 2011; Cassano et al. 2012).

In this context, we are studying the occurrence of radio halos in a sample of 75 galaxy clusters with $M_{500} \gtrsim 6 \times 10^{14} M_{\odot}$ and $z=0.08-0.33$ selected from the Planck SZ cluster catalogue (Planck Collaboration XXIX 2014). First results, based on a sub-sample of 57 clusters with available radio information, were published in Cuciti et al. (2015). We are completing the analysis of the sample using new Giant Metrewave Radio Telescope (GMRT) and Jansky Very Large Array (JVLA) data of all the clusters that lack deep radio observations in order to determine the possible presence of diffuse emission. This work led to the discovery of a number of cluster-scale radio sources. Here we present new GMRT and JVLA data of two clusters, $\mathrm{ZwCl}$ 0634.1+4750 (Z0634, hereafter) and Abell 1451 (A1451, hereafter), classified as candidate radio halos in Cuciti et al. (2015). Their properties are listed in Table 1.

Throughout this paper we assume a $\Lambda \mathrm{CDM}$ cosmology with $H_{0}=70 \mathrm{~km} \mathrm{~s}^{-1} \mathrm{Mpc}^{-1}, \Omega_{\Lambda}=0.7$ and $\Omega_{\mathrm{m}}=0.3$. With this cosmology $1^{\prime \prime}$ corresponds to a physical scale of $2.953 \mathrm{kpc}$ at the redshift of Z0634 and to $3.287 \mathrm{kpc}$ at the redshift of A1451.

\section{Radio observations and data analysis}

We performed the data reduction, both calibration and imaging, with the Common Astronomy Software Applications package (CASA; McMullin et al. 2007). An overview of the radio observations presented here is given in Table 2, where we report the name of the clusters, the interferometer and the observing frequency, the date of the observation, the flux calibrator, the phase calibrator, the beam and the rms noise achieved in the images made with the "Briggs" weighting scheme (robust=0). Throughout the paper, the error $\Delta_{\mathrm{S}}$ associated with a flux density measurement $S$ is calculated as:

$\Delta_{\mathrm{S}}=\sqrt{\left(\mathrm{rms} \times \sqrt{\frac{A_{\mathrm{s}}}{A_{\mathrm{b}}}}\right)^{2}+\left(\sigma_{\mathrm{c}} \times S\right)^{2}}$,

where $A_{\mathrm{s}}$ is the source area, $A_{\mathrm{b}}$ is the beam area and $\sigma_{\mathrm{c}}$ is the calibration uncertainty. Details about the observations and the data reduction are discussed in the following subsections.

\subsection{GMRT observations}

Z0634 and A1451 were observed with the GMRT at $320 \mathrm{MHz}$ using an observing bandwidth of $32 \mathrm{MHz}$ subdivided into 256 channels (project code: 28_066, P.I. V. Cuciti). The total time on source for each cluster is $\sim 5 \mathrm{~h}$ and they were observed with a duty cycle of $24 \mathrm{~min}$ on target and $6 \mathrm{~min}$ on the phase calibrator. The flux density calibrators were observed for $\sim 15 \mathrm{~min}$ at the beginning and at the end of the observations. The flux density scale was set following Scaife \& Heald (2012). The bandpass was corrected using the flux density calibrators. As a first step we obtained amplitude and gain corrections for the primary calibrator in ten central neighbour channels free of radiofrequency interference (RFI); these solutions were applied before determining the bandpass in order to remove possible time variations of the gains during the observation. Once we applied the bandpass, gain solutions for all the calibrator sources on the full range of channel were determined and transferred to the target source. Automatic removal of RFI was performed with the CASA task flagdata (mode $=r f l a g)$. The central 220 channels were averaged to 22 channels each $\sim 1.3 \mathrm{MHz}$ wide to reduce the size of the dataset without introducing significant bandwidth 
V. Cuciti et al.: New giant radio sources and underluminous radio halos in two galaxy clusters

Table 2. Radio observations.

\begin{tabular}{|c|c|c|c|c|c|c|}
\hline Name & Observation & Date & Flux cal & Phase cal & $\begin{array}{l}\text { Beam } \\
\text { (“X") }\end{array}$ & $\begin{array}{c}\text { rms noise } \\
(\mu \mathrm{Jy} / \mathrm{b})\end{array}$ \\
\hline Abell 1451 & $\begin{array}{c}\text { GMRT } 320 \mathrm{MHz}^{d} \\
\text { JVLA } L \text {-band DnC array }^{b} \\
\text { JVLA } L \text {-band BnA array }^{c}\end{array}$ & $\begin{array}{l}23 \text { May } 2015 \\
07 \text { Jan. } 2016 \\
18 \text { May } 2015 \\
\end{array}$ & $\begin{array}{c}3 \mathrm{C} 286 \\
3 \mathrm{C} 286 \\
3 \mathrm{C} 147,3 \mathrm{C} 286 \\
\end{array}$ & $\begin{array}{c}1130-148 \\
1130-148 \\
- \\
\end{array}$ & $\begin{array}{c}11.6 \times 8.1 \\
44.4 \times 17.1 \\
4.7 \times 3.3 \\
\end{array}$ & $\begin{array}{l}95 \\
60 \\
25 \\
\end{array}$ \\
\hline $\mathrm{ZwCl} 0634.1+4750$ & $\begin{array}{l}\text { GMRT } 320 \mathrm{MHz}^{a} \\
\text { JVLA } L \text {-band D array } \\
\text { JVLA } L \text {-band B array }\end{array}$ & $\begin{array}{l}19 \text { Dec } 2015 \\
19 \text { Oct. } 2015 \\
\text { 22 Feb. } 2015\end{array}$ & $\begin{array}{c}3 \mathrm{C} 147 \\
3 \mathrm{C} 147 \\
3 \mathrm{C} 147,3 \mathrm{C} 286\end{array}$ & $\begin{array}{c}3 \mathrm{C} 147 \\
\mathrm{~J} 0713+4349 \\
-\end{array}$ & $\begin{aligned} 9.7 & \times 7.3 \\
35.7 & \times 30.7 \\
3.5 & \times 3.3\end{aligned}$ & $\begin{array}{l}85 \\
45 \\
26\end{array}$ \\
\hline
\end{tabular}

Notes. ${ }^{(a)}$ obs No 8266 (project code 28_066), ${ }^{(b)}$ project code 15B-035, ${ }^{(c)}$ project code 15A-270, ${ }^{(d)}$ obs No 7798 (project code 28_066).

smearing within the primary beam. A number of phase-only selfcalibration rounds was carried out to reduce residual phase variations. Wide field imaging was implemented to account for the non-coplanarity of the baselines (wprojection) and wide band imaging (mode $=m f s$, nterms $=2$ ) was used to consider the frequency dependence of the sources in the sky. We applied further manual editing to the data, flagging bad self-calibration solutions and visually inspecting the residual $u v$-data. However, a number of bright sources in the field of view limited the dynamic range of the image, therefore we obtained direction dependent amplitude and phase solutions for these sources and then subtracted them out of the $u v$-data. This method is commonly referred to as "peeling" technique.

We used the "Briggs" weighting scheme with robust=0 throughout the self-calibration and we produced final "full-resolution" images with beam $\sim 10$ " and rms noise $\sim 0.1 \mathrm{mJy} /$ beam (see Table 2 for details). We then produced low-resolution images by using different weighting schemes and/or tapering down the long baselines. Images were corrected for the primary beam response. The residual calibration errors $\left(\sigma_{\mathrm{c}}\right.$ in Eq. (1)) are within $10 \%$ (e.g. Chandra et al. 2004).

\subsection{JVLA observations}

We carried out JVLA $L$-band observations of Z0634 in D array and of A1451 in DnC array (project code: 15B-035, P.I. V. Cuciti). The hybrid configuration DnC was used to obtain a roundish beam for targets with $\delta<-15^{\circ}$, such as A1451. The total bandwidth, from 1 to $2 \mathrm{GHz}$, is divided into 16 spectral windows, each with 64 channels of $2 \mathrm{MHz}$ in width. The total time on source for each cluster was $\sim 45 \mathrm{~min}$. We observed the phase calibrators for $\sim 2$ min every $\sim 20 \mathrm{~min}$. The flux density calibrators were observed for $\sim 5 \mathrm{~min}$ at the end of the observation.

In addition, these two clusters were observed with the JVLA in $L$-band in B array as part of a project mainly aimed at studying magnetic fields in galaxy clusters through Faraday rotation measurements of polarised background sources in the Planck ESZ galaxy cluster sample (project 15A-270, P.I. R. van Weeren). The hybrid configuration BnA was used for A1451, given its southern declination. Each cluster was observed for a total of $\sim 40 \mathrm{~min}$. Both 3C286 and 3C147 were observed as flux density calibrators, while no phase calibrator was observed in this project.

As a first step, the data were Hanning smoothed. We applied the pre-determined antenna position offset and elevationdependent gain tables. The flux density scale was set according to Perley \& Butler (2013). We determined amplitude and phase solutions for the flux calibrators in the ten central channels of each spectral window in order to remove possible time variations during the calibrator observation. These solutions were preapplied to find the delay terms (gaintype=' $\mathrm{K}$ ') and to correct for the bandpass response. We obtained the complex gain solutions for the calibrator sources (also for the phase calibrator, if present) on the full bandwidth pre-applying the bandpass and delay solutions. Finally, we applied all the calibration tables to the target field. Automatic RFI flagging was applied to the target field using the CASA task flagdata (mode=tfcrop). To reduce the size of the dataset, we averaged the 48 central channels of each spectral window to 6 channels and we averaged in time with a time bin of $15 \mathrm{~s}$.

We ran several rounds of phase-only self-calibration on the target field and a final amplitude and phase self-calibration. Manual flagging of additional data was applied by visually inspecting the solutions of the self-calibration. The wprojection algorithm was used to take into account the non-coplanar nature of the array. Wide band imaging is crucial when dealing with the $1 \mathrm{GHz}$ bandwidth of the JVLA, therefore we used at least two (sometimes three) Taylor terms (nterms $=2-3$ ) to take the frequency dependence of the brightness distribution into consideration. The imaging process involves the use of clean masks that have been made with the PyBDSM package (Mohan \& Rafferty 2015). For the self-calibration we used the "Briggs" weighting scheme with robust $=0$ and we made final "full-resolution" images, whose properties are listed in Table 2. Images were corrected for the primary beam attenuation. The absolute flux scale uncertainties $\left(\sigma_{\mathrm{c}}\right.$ in Eq. $\left.(1)\right)$ are assumed to be within $2.5 \%$ (Perley \& Butler 2013).

After a separate calibration and self-calibration, the B and D array observations were combined. Unfortunately the pointings did not coincide exactly and this severely affected the quality of the combined image, especially around bright sources away from the phase centre. In fact, the primary beam response at the position of those sources is different and this leads to considerable differences in their apparent fluxes between the two observations. To overcome this problem, we subtracted those sources from the $u v$-data and then we combined the datasets. In particular, we performed the subtraction of those sources separately for each spectral window. This is particularly important for sources that are close to the border of the primary beam because the size of the primary beam depends on the frequency, hence the flux density of those sources is considerably different in different spectral windows. The combined $\mathrm{B}+\mathrm{D}$ array images have a "full resolution" of $\sim 6$ ".

\section{A1451}

A1451 (alternative names: RXC J1203.2-2131, PSZ1 G288.26+39.94 $)$ is a massive $\left(M_{500}=7.32 \times 10^{14} M_{\odot}\right.$, Planck Collaboration XXIX 2014) and hot (13.4 keV, Valtchanov et al. 2002) galaxy cluster at redshift $z=0.199$. The meta-catalog of $\mathrm{X}$-ray detected clusters of galaxies (MCXC) reports an X-ray 


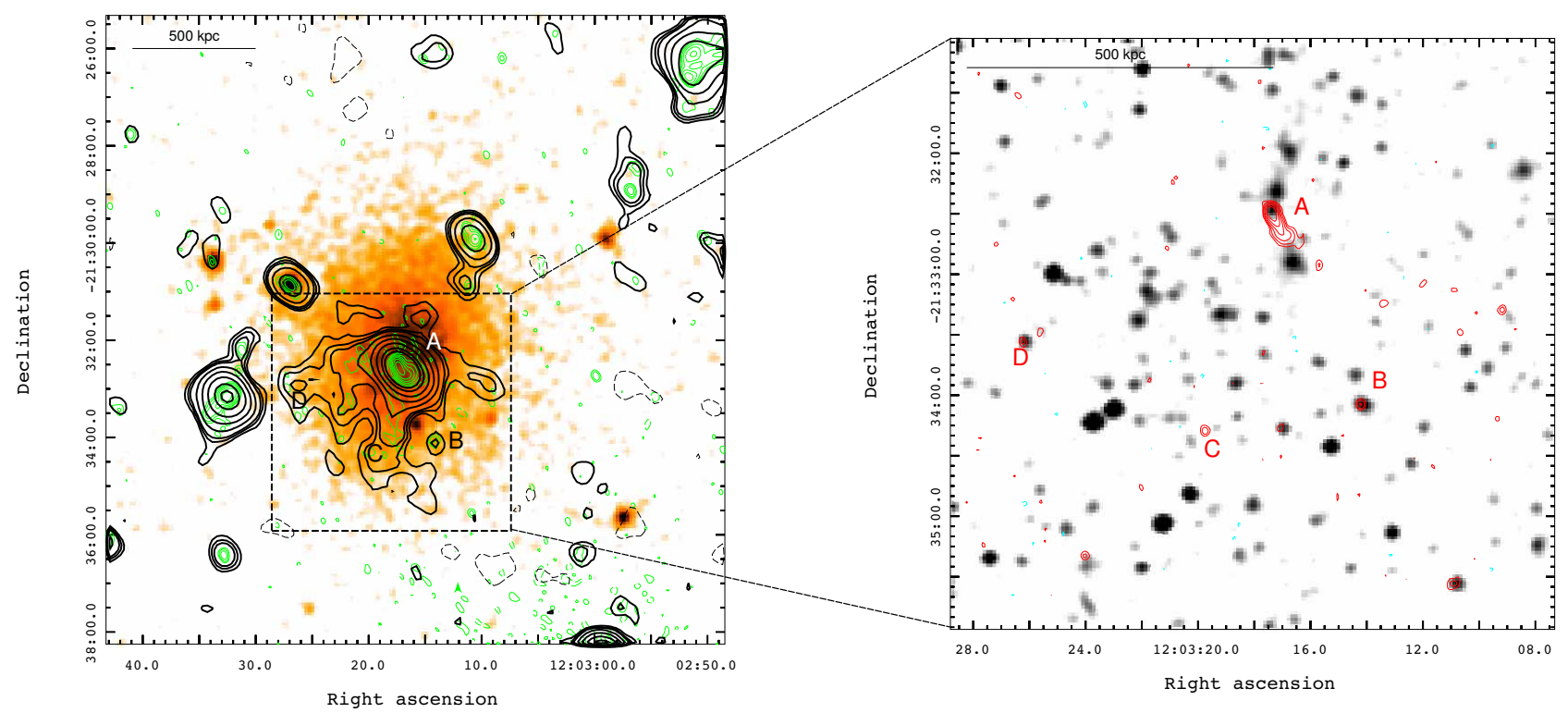

Fig. 1. Left: A1451 GMRT $320 \mathrm{MHz}$ full (green) and low (black) resolution contours superimposed on the X-ray XMM-Newton image. Lowresolution contour levels (black) are $(2,3,4,8,16,32 \ldots) \times \sigma$ with rms noise $\sigma=0.3 \mathrm{mJy} /$ beam and beam $=39.4^{\prime \prime} \times 27.9^{\prime \prime}$. Full-resolution contours start at $0.3 \mathrm{mJy} / \mathrm{beam}$ and are spaced by a factor 2 . The full-resolution beam is $11.6^{\prime \prime} \times 8.1^{\prime \prime}$ The first negative contours are dashed. Right: JVLA B array contours (red) of the central part of the cluster superimposed on the optical DSS image. Sources are labelled A to D as in Fig. 3. Contours start at $0.08 \mathrm{mJy} /$ beam and are spaced by a factor 2 . The beam is $4.7^{\prime \prime} \times 3.3^{\prime \prime}$. The first negative contour is shown with a cyan dashed line.

luminosity in the $[0.1-2.4] \mathrm{keV}$ band $L_{500}=6.61 \times 10^{44} L_{\odot}$ (Piffaretti et al. 2011).

Although the X-ray emission of A1451 (Figs. 1 and 3) is fairly regular, several pieces of evidence support the idea of ongoing merging activity for this cluster. Indeed, the high temperature and velocity dispersion are significantly in excess with respect to the estimates derived from weak lensing data (Cypriano et al. 2004). Moreover, both the X-ray morphology and the mass distribution derived using the distortions of the faint background galaxies (Cypriano et al. 2004) are elongated in the North-South direction. No radio diffuse emission has been detected in A1451 with ATCA $1.4 \mathrm{GHz}$ observations (Valtchanov et al. 2002). On the other hand, hints of diffuse emission have been detected by Cuciti et al. (2015) in their reanalysis of the NVSS $u v$-dataset (Condon et al. 1998).

Here we present the discovery of a faint radio halo at the centre of the cluster A1451 and a possible radio relic using a deep GMRT observation at $320 \mathrm{MHz}$ and JVLA observations at $1.5 \mathrm{GHz}$. These sources are discussed in detail below. Their properties are summarised in Table 3.

\subsection{Radio halo}

A number of discrete radio sources are visible on the cluster area in the GMRT $320 \mathrm{MHz}$ full-resolution image (labelled A to D in Fig. 1). In particular, there is a radio galaxy at the centre of the cluster (source A), with a total flux density of $\sim 80 \mathrm{mJy}$ at $320 \mathrm{MHz}$ and $\sim 17 \mathrm{mJy}$ at $1.5 \mathrm{GHz}$. The JVLA B array image (Fig. 1, right panel) shows that the brighter part of this source is followed by a short, low-surface-brightness tail that makes its subtraction from the $u v$-data difficult. In addition to the individual sources, some positive residuals are visible in the fullresolution GMRT $320 \mathrm{MHz}$ image (green contours in Fig. 1, left panel). The low-resolution image (black contours in Fig. 1, left panel) clearly shows the presence of extended emission, that we classify as a radio halo. The radio halo has a largest angular size (LAS) of $\sim 4$ arcmin in the N-S direction, corresponding to a projected largest linear size (LLS) of $\sim 750 \mathrm{kpc}$. It is mainly

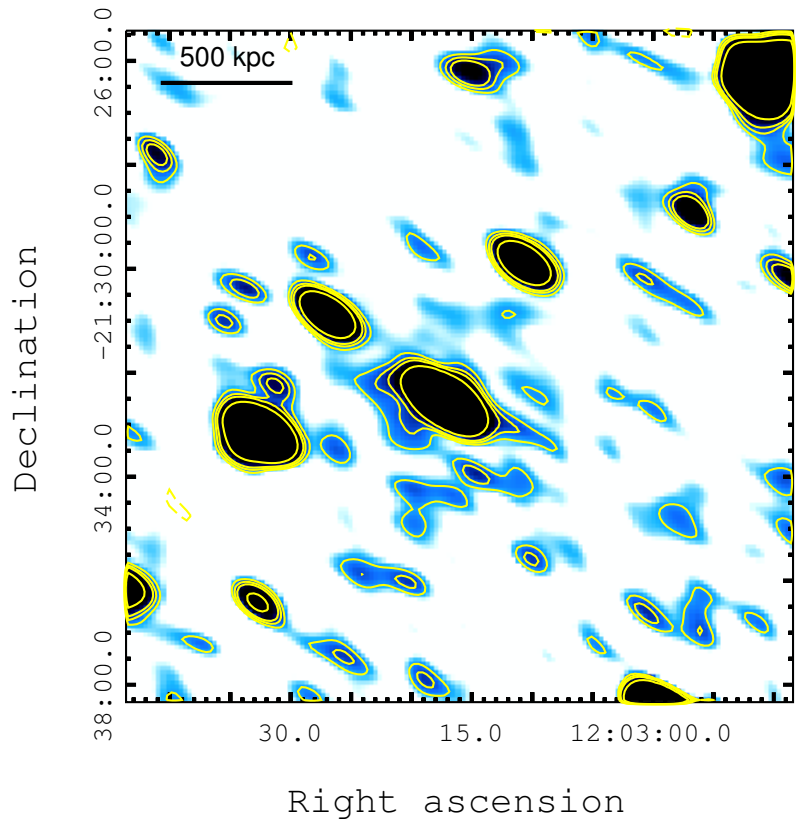

Fig. 2. JVLA D array colour image and contours of A1451. The contour levels are $(2,3,4,8,16,32 \ldots) \times \sigma_{\text {rms }}$ with rms noise $\sigma_{\mathrm{rms}}=60 \mu \mathrm{Jy} / \mathrm{beam}$ and beam $=44.4^{\prime \prime} \times 17.1^{\prime \prime}$. The first negative contour is dashed.

extended towards the Southern part of the cluster. There are hints of diffuse emission also in the Northern part, both in the GMRT and JVLA images (Fig. 2), but their surface brightness is below twice the rms noise level of the images. We measured the flux density of the radio halo on the low-resolution image (Fig. 1, left panel), then we subtracted the flux density of the central radio galaxy and of the three point-like sources. Specifically, we measured the flux density of the point-like sources on the fullresolution $\left(\sim 10^{\prime \prime}\right)$ image, while we measured the flux density of source A on the low-resolution image, to take into account also its extended emission. We obtained a flux density for the 
radio halo of $S_{320 \mathrm{MHz}} \sim 22 \mathrm{mJy}$. It is likely that while subtracting source $\mathrm{A}$, we are also subtracting part of the radio halo flux density. We measured the mean surface brightness of the central part of the radio halo (around source A) and we estimated that in the area of source $\mathrm{A}$ there are $\sim 5 \mathrm{mJy}$ of radio halo flux density, leading to a total flux density of the radio halo of $S_{320 \mathrm{MHz}}=27.3 \pm 2.3 \mathrm{mJy}$.

An equivalent procedure has been applied to measure the flux density of the radio halo at $1.5 \mathrm{GHz}$, that is, we measured the radio flux density on the whole region of the halo from the D array image (Fig. 2, resolution $\sim 40^{\prime \prime}$ ) and we subtracted the flux density of the central source, measured on the same map, and of the three point-like sources, taken from the B array image (resolution $\sim 4^{\prime \prime}$ ). The flux density of the radio halo is $S_{1.5 \mathrm{GHz}}=5.0 \pm 0.5 \mathrm{mJy}$, which corresponds to a radio power $P_{1.4 \mathrm{GHz}}=6.4 \pm 0.7 \times 10^{23} \mathrm{~W} / \mathrm{Hz}$, assuming a spectral index $\alpha=-1.2$ for the $k$-correction. These values take into account the portion of the radio halo covered by source A.

To evaluate the spectrum of the halo between 320 and $1500 \mathrm{MHz}$ (B+D array JVLA combined observations) we produced images with uniform weighting, using the common $u v$ range and tapering down to the same resolution $\left(\sim 20^{\prime \prime}\right)$ and we measured the flux density of the radio halo on exactly the same region. We performed the imaging using two approaches: i) cleaning only inside the mask containing all the sources in the field until the residuals were approximately two times the rms noise of the image or ii) after that, cleaning also on the rest of the field down to two times the rms noise. Depending on how we cleaned the images and on the region where we measure the flux density of the radio halo we found values for the spectral index in the range $-1.3 \lesssim \alpha \lesssim-1.1$. In particular, we found the steepest spectral indices using approach (ii). We note that a higher cleaning threshold does not yield different results; indeed, if we clean until the residuals are approximately three times the rms noise of the images, we obtain no significant difference in the flux density of the radio halo at both frequencies and using both approaches (i) and (ii).

\subsection{A distant relic?}

We detected an elongated diffuse source located $\sim 15$ arcmin North of the galaxy cluster centre (Fig. 3), both at $320 \mathrm{MHz}$ and in the JVLA D array $1.5 \mathrm{GHz}$ image (Fig. 4). Based on its morphology and location we classify it as a candidate radio relic. A zoom on the candidate relic, superimposed on the optical Digitized Sky Survey (DSS) image, is shown in Fig. 4. If assumed to be at the redshift of the cluster, this source is $\sim 3 \mathrm{Mpc}$ away from the cluster centre. Its LAS in the E-W direction is $\sim 6.5$ arcmin, which would correspond to a LLS $\sim 1.3 \mathrm{Mpc}$. A point-like radio source with a clear optical counterpart (labelled S in Fig. 4) is embedded in the diffuse emission. With the same procedure used for the radio halo, we measured the flux density of the point-like source from high-resolution images and we subtracted it from the flux density measured on the candidate relic area, obtaining a flux density of $35.5 \pm 5.3 \mathrm{mJy}$ at $320 \mathrm{MHz}$ and $9.0 \pm 0.5 \mathrm{mJy}$ at $1.5 \mathrm{GHz}$.

To evaluate the spectral index of the candidate radio relic between $320 \mathrm{MHz}$ and $1.5 \mathrm{GHz}$ (D array observation), we used uniform weighting, the common $u v$-range and we convolved the images to the same resolution $\left(50^{\prime \prime}\right)$. The integrated spectral index of this source is $\alpha=-1.1 \pm 0.1$. The resolution of these observations does not allow a reliable analysis of a possible spectral index gradient along the $\mathrm{N}-\mathrm{S}$ axis. Conversely, the data allow us to conclude that there is no evidence of a spectral in-

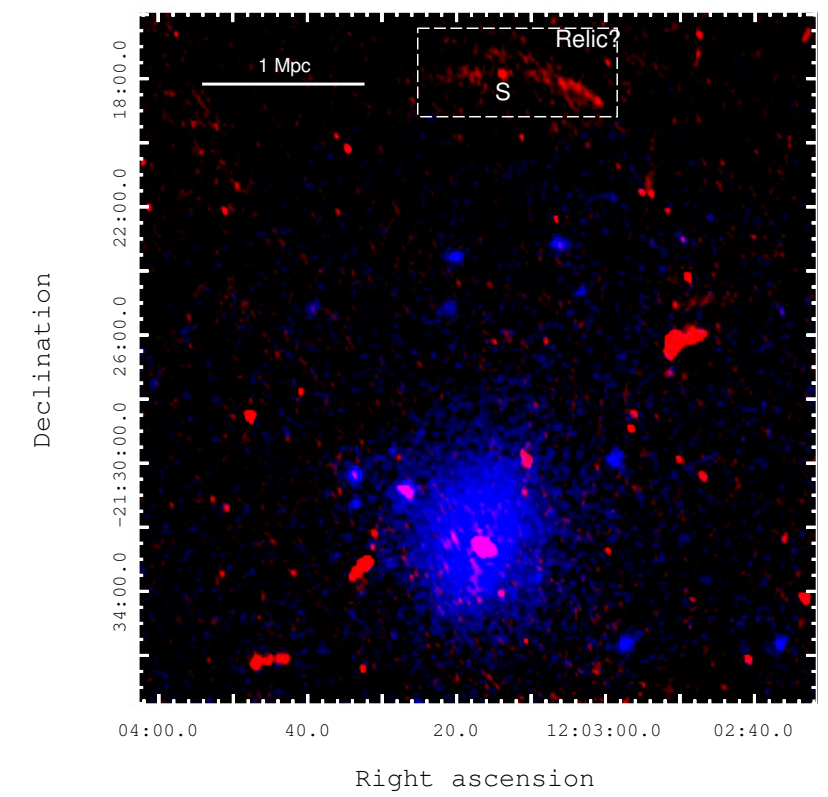

Fig. 3. A1451 GMRT $320 \mathrm{MHz}$ image (red) superimposed on the X-ray XMM-Newton image (blue).

dex gradient along the E-W axis, as expected in the case of an active source in $\mathrm{S}$ refurbishing the extended emission. The radio power of the candidate relic at $1.4 \mathrm{GHz}$ is $P_{1.4 \mathrm{GHz}}=$ $1.13 \pm 0.06 \times 10^{24} \mathrm{~W} / \mathrm{Hz}$, assuming a spectral index $\alpha=-1.1$ for the $k$-correction.

\section{Zwcl0634.1+4747}

Little is known in the literature about Zwcl0634.1+4747 (alternative names: PSZ1 G167.64+17.63, CIZA J0638.1+4747, RX J0638.1+4747). It is at redshift $z=0.174$ and the MCXC catalogue reports an X-ray luminosity in the [0.1-2.4] keV band $L_{500}=4.72 \times 10^{44} L_{\odot}($ Piffaretti et al. 2011). The mass within $R_{500}$ estimated by Planck Collaboration XXIX (2014) is $M_{500}=6.52 \times 10^{14} M_{\odot}$. An X-ray Chandra archival observation of Z0634 has been analysed in Cuciti et al. (2015) where evidence of a non-relaxed dynamical state has been found. Indeed the Chandra image (Fig. 5, left panel) shows the presence of substructures in the X-ray surface brightness distribution and the morphology of the cluster is elongated in the East-West direction.

The JVLA D array image of Z0634 is shown in Fig. 5 (left panel). The cluster hosts diffuse, centrally located radio emission that we classify as a radio halo. Two head-tail (HT) radio galaxies are present in the cluster field, one located North of the radio halo (labelled HT in Fig. 5) and one located at the $\mathrm{S}-\mathrm{E}$ edge of the cluster (source $\mathrm{C}$ in Fig. 5). While source $\mathrm{C}$ has the typical morphology and spectral properties of many HT radio galaxies found in clusters (e.g. O'Dea \& Owen 1986; Giacintucci \& Venturi 2009; Owen et al. 2014), the radio galaxy named HT has some unusual characteristics. The radio halo and the HT are described in the following subsections. Their properties are summarised in Table 3.

\subsection{Radio halo}

The radio halo in Z0634 extends over $\sim 600 \mathrm{kpc}$ in the E-W direction and follows the morphology of the X-ray emission of the cluster (Fig. 5, left panel). The two HT radio galaxies, HT and C, are difficult to subtract from the $u v$-data, thus we measured the radio halo flux density on an area that avoids these two sources. 

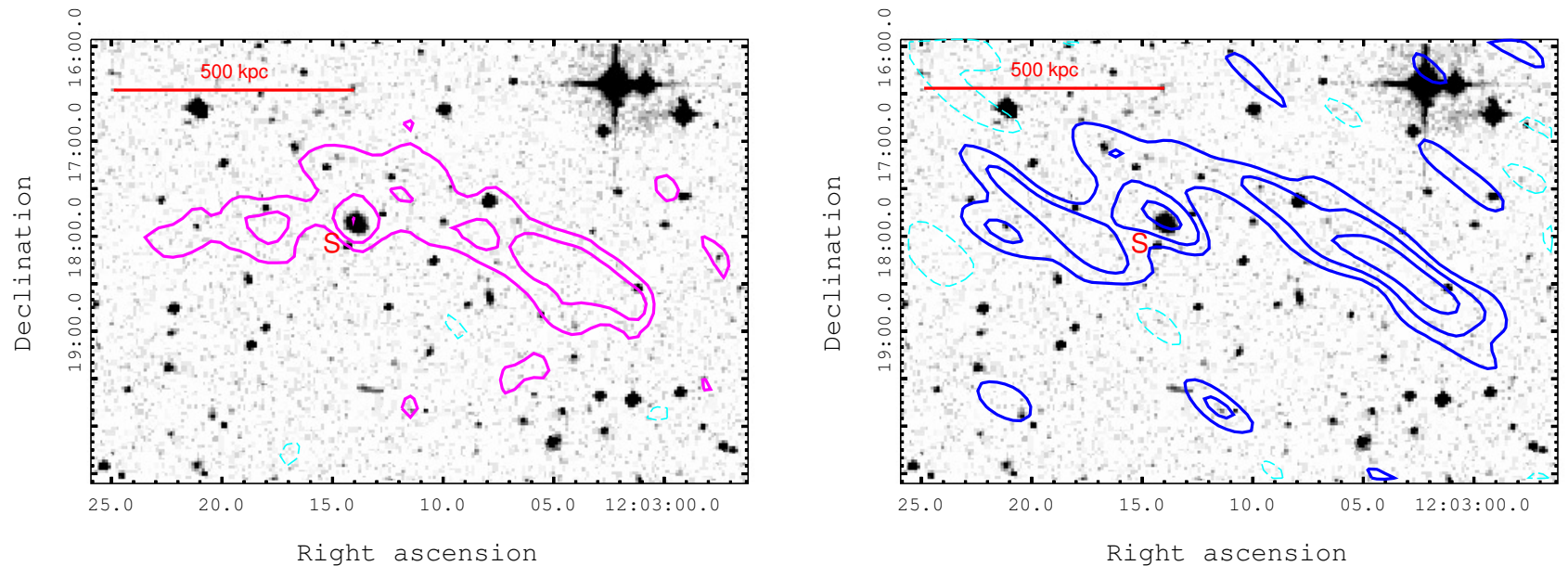

Fig. 4. GMRT $320 \mathrm{MHz}$ (left) and JVLA D array $1.5 \mathrm{GHz}$ (right) contours of the relic in A1451 superimposed on the optical DSS image. Left: radio contours starts at $0.6 \mathrm{mJy} / \mathrm{beam}$ and are spaced by a factor 2 . The first negative contour is shown with a cyan dashed line. The rms noise of the radio image is $\sim 0.2 \mathrm{mJy} /$ beam with a beam of $23.3^{\prime \prime} \times 18.0^{\prime \prime}$. Right: radio contours starts at $0.18 \mathrm{mJy} / \mathrm{beam}$ and are spaced by a factor 2 . The first negative contour is shown with a cyan dashed line. The rms noise of the radio image is $\sim 60 \mu \mathrm{Jy} / \mathrm{beam}$ with a beam of $44.4^{\prime \prime} \times 17.1^{\prime \prime}$.
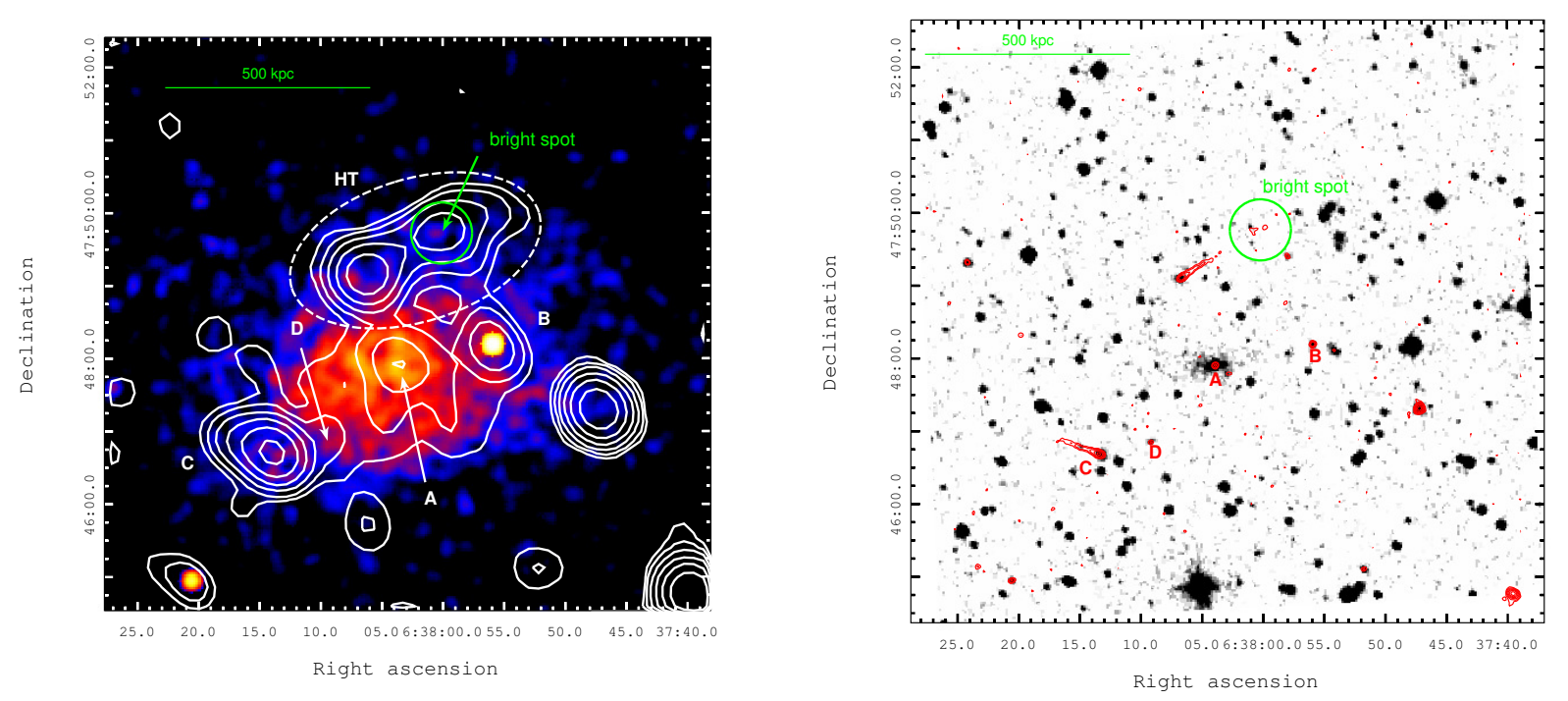

Fig. 5. Left: Z0634 JVLA D array $1.5 \mathrm{GHz}$ contours (white) superimposed on the X-ray Chandra image. Radio contours starts at $0.12 \mathrm{mJy} / \mathrm{beam}$ and are spaced by a factor 2 . The rms noise of the radio image is $\sim 45 \mu \mathrm{Jy} /$ beam with a beam of $35.7^{\prime \prime} \times 30.7^{\prime \prime}$. Right: JVLA B array contours (red) superimposed on the optical DSS image. Contours starts at $0.09 \mathrm{mJy} /$ beam and are spaced by a factor 2 . The beam is $3.5^{\prime \prime} \times 3.3^{\prime \prime}$.

Still, there are three sources (labelled A, B and D in Fig. 5) embedded in the radio halo emission. The high-resolution $\left(\sim 3^{\prime \prime}\right)$ JVLA B array image (Fig. 5, right panel) reveals that they are point-like sources, so their flux density, measured from the B array image $(\sim 1.4 \mathrm{mJy}$ in total), can be safely subtracted from the measurement on the radio halo region. We obtained a flux density of the radio halo $S_{1.5 \mathrm{GHz}}=3.3 \pm 0.2 \mathrm{mJy}$, corresponding to a radio power $P_{1.4 \mathrm{GHz}}=3.1 \pm 0.2 \times 10^{23} \mathrm{~W} / \mathrm{Hz}$, assuming a spectral index $\alpha=-1.2$ for the $k$-correction.

The flux density of the radio halo at $320 \mathrm{MHz}$, after the subtraction of the point sources mentioned above, is $S_{320 \mathrm{MHz}}=$ $20.3 \pm 2.7 \mathrm{mJy}$. We note that in the low-resolution GMRT $320 \mathrm{MHz}$ image (Fig. 6) the radio halo does not extend to the HT and source $\mathrm{C}$, thus we can reasonably exclude that we have lost a substantial part of the radio halo emission by avoiding those regions in the measurement of the radio halo flux density. To evaluate the spectral index of the radio halo, we produced images at 320 and $1500 \mathrm{MHz}(\mathrm{B}+\mathrm{D}$ array JVLA observations) with uniform weighting and using the same $u v$-range and resolution $\left(\sim 20^{\prime \prime}\right)$ and we measured the flux density of the radio halo on the same area. As done for A1451, we performed the imaging with two approaches: i) cleaning only inside the mask that contains all the sources in the field or ii) after that, cleaning also on the rest of the field down to two times the rms noise. We found that the spectral index of the radio halo ranges from -1 to -1.3 , depending on where we measure the flux densities and how we clean the images ( $\alpha$ is steeper in case (ii)). We obtained similar results setting the threshold at three times the rms noise in the cleaning process.

\subsection{Head tail}

The "head" of the HT radio galaxy is located $\sim 110^{\prime}$ NE from the cluster X-ray centroid (i.e. at a projected distance of $\sim 330 \mathrm{kpc}$ ). 
V. Cuciti et al.: New giant radio sources and underluminous radio halos in two galaxy clusters

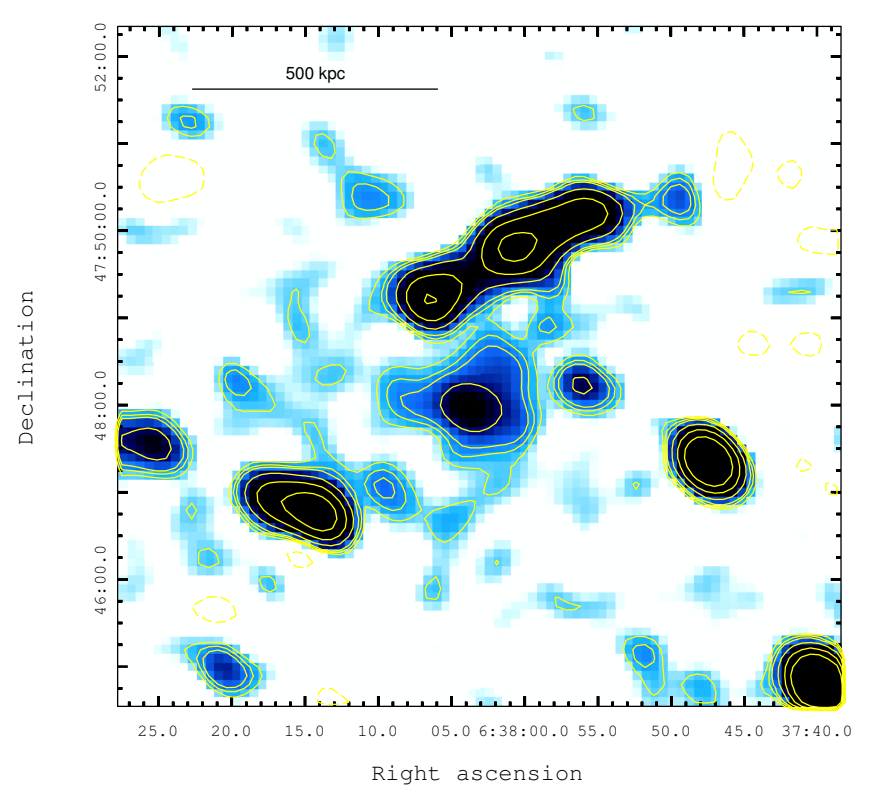

Fig. 6. GMRT $330 \mathrm{MHz}$ colour image and contours of Z0634. The contour levels are $(2,3,4,8,16,32 \ldots) \times \sigma_{\text {rms }}$ with $\sigma_{\text {rms }}=0.25 \mathrm{mJy} / \mathrm{beam}$. The resolution is $30.5^{\prime \prime} \times 23.0^{\prime \prime}$. The first negative contour is dashed.

This bright nucleus is followed by a lower-surface-brightness tail that extends in the SE-NW direction (Fig. 5). Considering the $3 \sigma$ contours of the GMRT $320 \mathrm{MHz}$ image (shown in Fig. 8), the angular extension of the HT is $\sim 3.5$ arcmin, corresponding to $\sim 600 \mathrm{kpc}$ at the cluster redshift (Fig. 7, left panel). Its linear extension decreases to $\sim 400 \mathrm{kpc}$ at $1.5 \mathrm{GHz}$ (Fig. 7, right panel), measured from the $3 \sigma$ contours of the $\mathrm{B}+\mathrm{D}$ array image. The total flux density of the HT at $320 \mathrm{MHz}$ is $42.5 \pm 4.3 \mathrm{mJy}$, while it is $8.9 \pm 0.3 \mathrm{mJy}$ at $1.5 \mathrm{GHz}$. The radio surface brightness does not decrease regularly along the tail. There is a spot of higher surface brightness, roughly at half length, visible at both frequencies. After this spot, on the Western side, the tail is brighter than before the spot (Fig. 7).

To make the spectral index map of the HT we used the spectral windows 6 and 7 of the JVLA B+D dataset and we produced JVLA and GMRT images with uniform weighting and common $u v$-range and convolved them to the same resolution $\left(15^{\prime \prime}\right)$. We blanked those pixels that in the GMRT $320 \mathrm{MHz}$ image have values below two times the rms noise $(\sim 0.25 \mathrm{mJy} /$ beam $)$ in order to take into account the extension of the tail at low frequency as much as possible. However, given the poor signal-to-noise ratio and the large errors associated with the spectral index (Fig. 8), we will not consider the westernmost part of the tail in the analysis in Sect. 5.4. As shown in Fig. 8, we found that the spectral index between $320 \mathrm{MHz}$ and $1.5 \mathrm{GHz}$ steepens with the distance from the nucleus, from $\alpha \sim-0.6$ to $\alpha \sim-2.5$. We also note that the bright spot is steeper that the Eastern (initial) part of the tail.

\section{Discussion}

\subsection{Testing the reliability of the spectral indices of the radio halos}

Compared to the current radio power-mass scaling relations (Cassano et al. 2013; Martinez Aviles et al. 2016), the radio halos in A1451 and Z0634 are underluminous, being 6-7 times below the correlation (Fig. 9). Since these radio halos are very faint, a reliable characterisation of their morphology and spectrum is challenging with the current observations. Here we investigate the possibility that the measurement of these spectral indices is biased because of the different capability of our GMRT and JVLA observations to properly recover such extended faint radio emission. For this purpose, we used the method of injecting fake radio halos into the datasets. This technique has been used in the literature to place upper limits to the diffuse radio flux density of clusters in which a radio halo is not detected (Brunetti et al. 2007; Venturi et al. 2008; Russell et al. 2011; Kale et al. 2013, 2015). In fact, an important outcome of those studies is that the flux of the fake radio halos is at least partly lost and this effect becomes more important for faint flux densities (e.g. Venturi et al. 2008). Therefore this warns that part of the flux of the radio halos in A1451 and Z0634 may not be properly recovered by our GMRT and JVLA observations.

We modelled the radio halo brightness profile with an exponential law in the form:

$I(r)=I_{0} \mathrm{e}^{-\frac{r}{r_{\mathrm{e}}}}$,

where $I_{0}$ is the central surface brightness and $r_{\mathrm{e}}$ is the $e$ folding radius (Orrú et al. 2007; Murgia et al. 2009). From the radio power-radio halo radius correlation (Eq. (6) in Cassano et al. 2007) we calculated the reference radii of the radio halos, $r_{h}$, assuming that they follow the radio power-mass correlation (Eq. (14) in Cassano et al. 2013). At first, we used $r_{\mathrm{e}}=r_{h} / 2.6$, where 2.6 is the median value of the quantity $r_{h} / r_{\mathrm{e}}$ for the radio halos studied by both Murgia et al. (2009) and Cassano et al. (2007), then we slightly change the value of $r_{\mathrm{e}}$ in the injected halos to reproduce the extension of the halos observed at the cluster centre. In the injected models, the radio halo profile was set to zero for $r>5 r_{\mathrm{e}}$.

For each cluster, we chose a region in the image void of bright sources and clear noise pattern and we created a set of fake radio halos with different integrated flux densities, centred on that region. We started injecting a fake radio halo that would lie on the radio power-mass correlation (Cassano et al. 2013) and we reduced the injected flux density until the recovered flux of the fake radio halo was similar to that of the radio halo that we observe at the cluster centre. Each model has been Fourier transformed into the MODEL_DATA column of the dataset including the wprojection algorithm to take into account the large field of view. We added the fake radio halo to the original visibilities and we imaged the modified dataset. We measured the recovered flux density of the fake radio halo and we compared it with the injected flux. Since the main aim of these injections is to check the reliability of the measured spectral indices of the two radio halos presented here, the images have been done minimising the differences between the two datasets as much as possible.

In particular, we used the common $u v$-range and we tapered down to the same resolution $\left(\sim 20^{\prime \prime}\right)$, as done in Sects. 3.1 and 4.1. We also used uniform weighting to minimise the difference in the $u v$-sampling. We performed the imaging with two approaches: (i) cleaning only inside the mask containing all the sources or (ii) cleaning inside the mask and also cleaning deeply on the rest of the field. In general, we found that the rms noise of the final image improves in case (ii), but the recovered flux of the injected radio halos is significantly lower (from 10 to $40 \%$ ) than in case (i), such effect being more prominent for faint injected flux densities.

An important outcome of this analysis is that, for both clusters, if we inject the flux density of a radio halo that lies on the radio luminosity-mass correlation, both interferometers are able to recover the great majority of its flux density. However, when reducing the injected flux, in order to match the observed one, the fraction that is recovered decreases. The decline of the recovered 

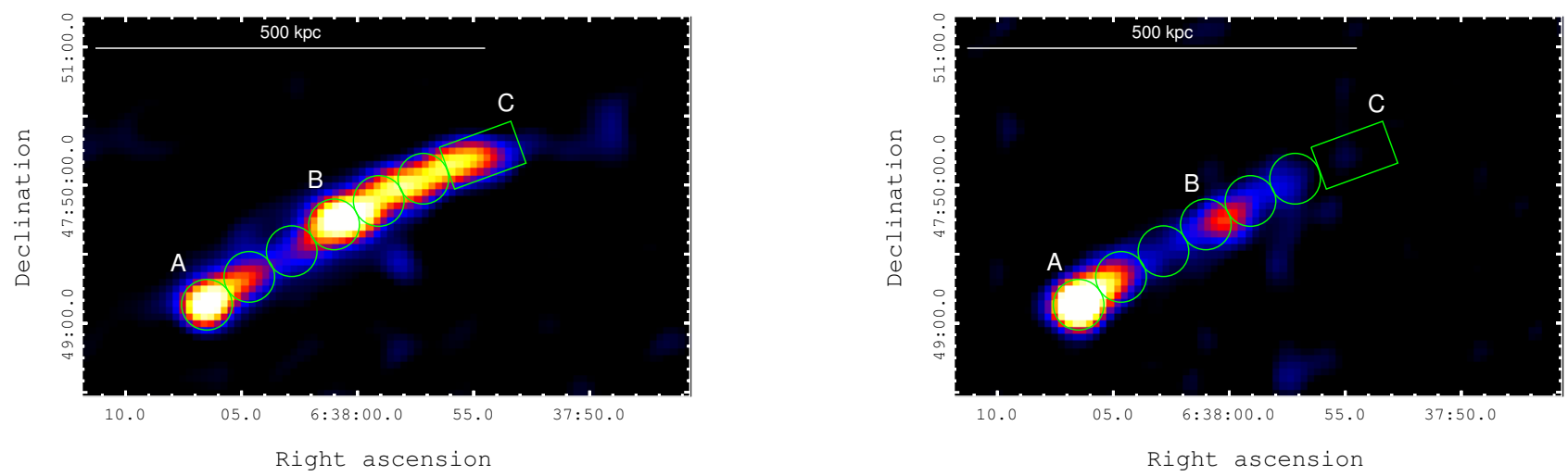

Fig. 7. GMRT $320 \mathrm{MHz}$ (left) and JVLA B+D array $1.5 \mathrm{GHz}($ right $)$ colour images. Both are convolved with a $15^{\prime \prime} \times 15^{\prime \prime}$ beam. The green regions mark the areas where we calculated the spectral index to trace its evolution along the tail.
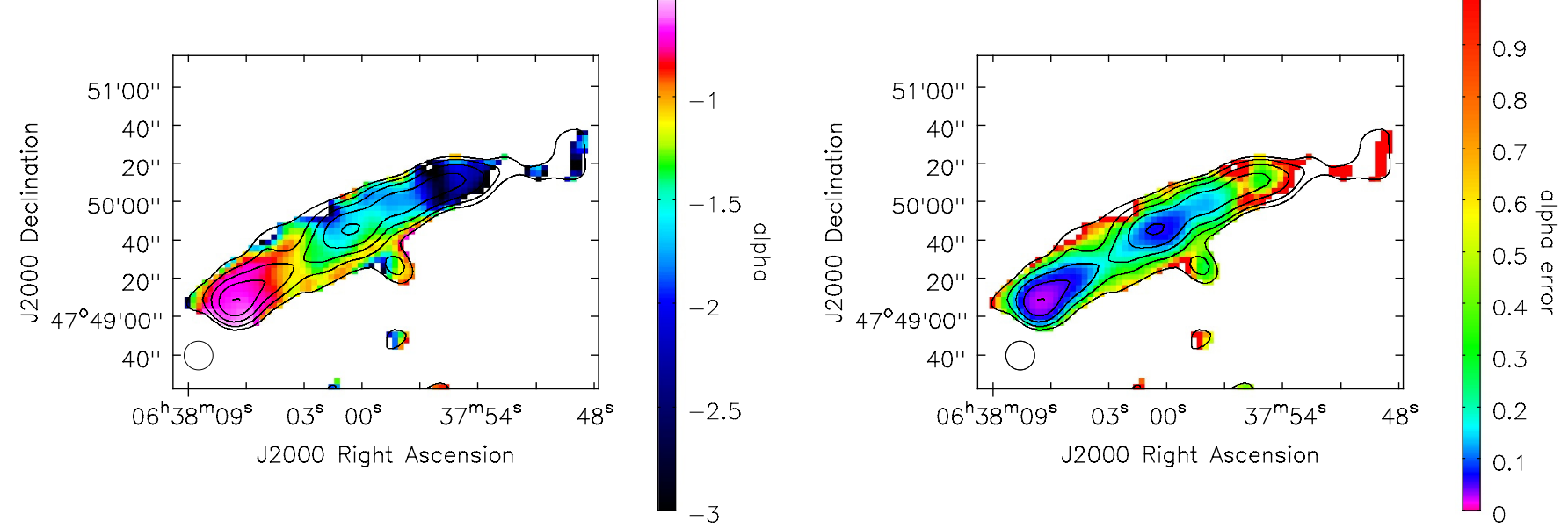

Fig. 8. Spectral index map (left) and associated spectral index error map (right) of the HT in Z0634 between $320 \mathrm{MHz}$ and $1.5 \mathrm{GHz}$ at resolution $15^{\prime \prime} \times 15^{\prime \prime}$. GMRT $320 \mathrm{MHZ}$ contours of the image used to make the spectral index map are drawn in black. Contours levels start from $0.42 \mathrm{mJy} /$ beam and are spaced by a factor 2 .

Table 3. Properties of the extended sources.

\begin{tabular}{ccccccc}
\hline \hline Name & Detection & $\begin{array}{c}S_{320 \mathrm{MHz}} \\
(\mathrm{mJy})\end{array}$ & $\begin{array}{c}S_{1.5 \mathrm{GHz}} \\
(\mathrm{mJy})\end{array}$ & $\begin{array}{c}P_{1.4 \mathrm{GHz}} \\
\left(10^{23} \mathrm{~W} / \mathrm{Hz}\right)\end{array}$ & Integrated $\alpha$ & $\begin{array}{c}\text { LAS } \\
(')\end{array}$ \\
\hline \multirow{2}{*}{$\mathrm{A} 1451$} & $\mathrm{RH}$ & $27.3 \pm 2.3$ & $5.0 \pm 0.6$ & $6.4 \pm 0.7$ & $>-1.3^{*}$ & 4.2 \\
& RR & $35.5 \pm 5.3$ & $9.0 \pm 0.5$ & $11.3 \pm 0.6$ & $-1.1 \pm 0.1$ & 6.5 \\
\hline \multirow{2}{*}{ Z0634 } & RH & $20.3 \pm 2.7$ & $3.3 \pm 0.2$ & $3.1 \pm 0.2$ & $>-1.3^{* *}$ & 3.3 \\
& HT & $42.5 \pm 4.3$ & $8.9 \pm 0.3$ & $8.5 \pm 0.3$ & $-1.25 \pm 0.08$ & 3.5 \\
\hline
\end{tabular}

Notes. Column 1: cluster name; Col. 2: detected source; Col. 3: flux density at $320 \mathrm{MHz}$; Col. 4: flux density at $1.5 \mathrm{GHz}$; Col. $5 \mathrm{radio}$ power at 1.4 GHz; Col. 6: integrated spectral index between $320 \mathrm{MHz}$ and $1.5 \mathrm{GHz}$. * See Sects. 3.1 and 5.1. ** See Sects. 4.1 and 5.1.

flux depends on a combination of sensitivity of the observations, density of the inner $u v$-coverage and image fidelity of the dataset (Venturi et al. 2008; Kale et al. 2013, 2015). More specifically, we found that our GMRT observations generally recover a higher fraction of the flux density of faint injected radio halos, assuming a spectral index $\alpha \leq-1.2$. This different performance can be explained by the different inner $u v$-coverage. Indeed, the full-track GMRT observation ensures a denser $u v$-sampling with respect to the snapshot JVLA observation (Fig. 10). For both A1451 and Z0634, in order to recover in the fake halo the same flux density that we find in the halo at the cluster centre at both frequencies, we need to inject a radio halo with a spectral index $\alpha \approx-1$. Unfortunately, a fully reliable estimate of the spectral index of these radio halos is prevented by the fact that the flux density of such faint diffuse emission can change significantly depending on where we measure it and how we perform the imaging (see Sects. 3.1 and 4.1). Nevertheless, the analysis based on the injections allows us to exclude the presence of radio halos with spectra significantly steeper than $\alpha \approx-1.3$ in A1451 and Z0634.

The results of the injections suggest that, even in the worst case, the flux of the radio halos can be underestimated at most by a factor of 1.8 (A1451) and 2.2 (Z0634) at $1.5 \mathrm{GHz}$, meaning that both the radio halos would be still $4-5$ times below the $P_{1.4 \mathrm{GHz}}-M_{500}$ correlation. We also note that at these flux levels, the morphology of the injected halos that is recovered from the maps may differ from that of the injected models. For this reason it is impossible to firmly establish weather the halo in A1451 extends only in the Southern region or if there is some emission 


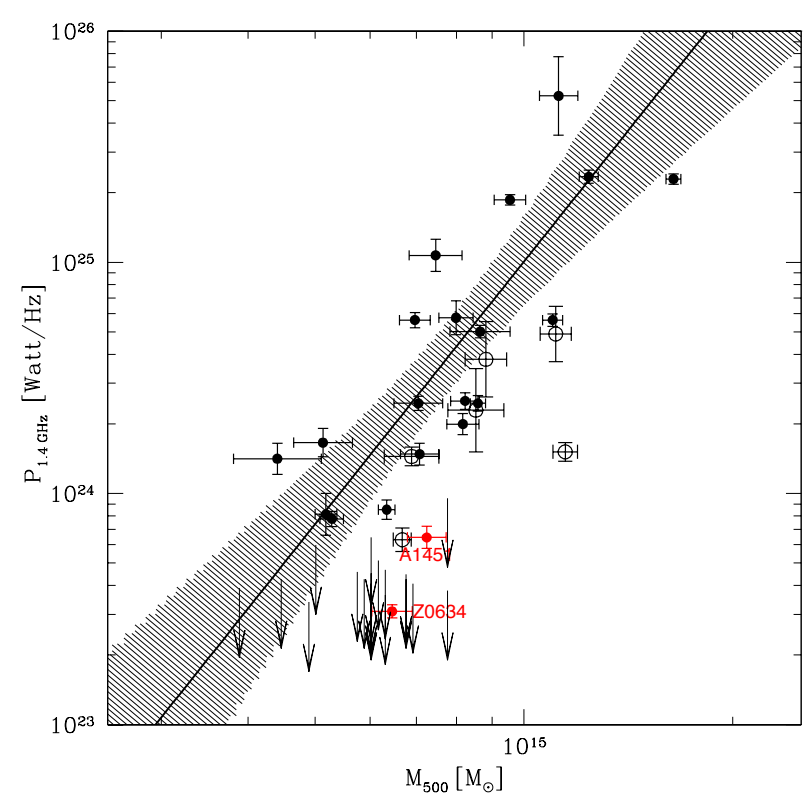

Fig. 9. Distribution of clusters in the $P_{1.4 \mathrm{GHz}}-M_{500}$ diagram. Different symbols indicate giant radio halos (black filled dots), ultra steep spectrum radio halos (black empty dots) and upper limits (black arrows) belonging to the Cassano et al. (2013) sample. The red dots represent the two clusters presented in this paper. The best fit relation for giant radio halos (black line) and the 95\% confidence level (shadowed region) from Cassano et al. (2013) are shown.

extending also in the Northern part. Finally, the injection procedure allows us to estimate the size of halos: we found that injected halo models with $r_{\mathrm{e}} \sim 140 \mathrm{kpc}$ and $r_{\mathrm{e}} \sim 130 \mathrm{kpc}$ (Eq. (2)) match the observed extension of the radio halos in A1451 and Z0634 when the recovered fluxes match the observed ones.

\subsection{Comparison with the current $P_{1.4 \mathrm{GHz}}-M_{500}$ correlation for radio halos}

Using statistical samples of 30-40 galaxy clusters, it has been shown that their radio properties on the Mpc scale have a bimodal distribution: either they host giant radio halos following the correlation between the cluster mass (or X-ray luminosity) and the radio luminosity, or they do not show diffuse radio emission at the sensitivity level of current observations (e.g. Cassano et al. 2013). This bimodality is connected to the dynamical state of clusters, with radio halo clusters undergoing merging events and non-radio halo clusters being generally relaxed. Since its discovery, it was proposed that a bimodal segregation results from the fact that the period of time that clusters spend in the region between the limits and the correlation is smaller than the time they spend on the correlation or in a much fainter state (e.g. Brunetti et al. 2009; Donnert et al. 2013). In general, this implies that the increase of the statistics would lead to the discovery of an increasing number of underluminous radio halos, sitting in the region between the correlation and the upper limits, which essentially sets the sensitivity of current observations. For illustrative purposes, we show in Fig. 9 the position of A1451 and Z0634 compared to the 95\% confidence level region filled by giant radio halos in Cassano et al. (2013). In order to detect underluminous radio halos, very deep observations of large samples of galaxy clusters are needed. Our sample of 75 clusters with $M_{500} \gtrsim 6 \times 10^{14} M_{\odot}$ and $z=0.08-0.33$ (Sect. 1) allows a unique statistical assessment of the distribution of clusters in the radio power-mass diagram and of the occurrence of radio halos in clusters. Here we anticipate that 30 radio halos are found in this sample; 7 of them sit in the region between the current radiomass correlations and the upper limits (Cuciti et al., in prep.). Although these underluminous halos are statistically subdominant, their presence confirms that the increase of the statistics allows to populate the region below the correlation in the radio-mass diagram, which also leads to an apparent increase of the scatter of such correlation toward lower luminosities. In fact there are reasons to expect a fairly large scatter in the scaling relation between the radio halo power and the cluster masses, due to the superposition of different intermediate radio halo states that are generated by the complex hierarchy of merger events.

One possibility proposed in the literature for underluminous radio halos is that they may have ultra steep spectra, resulting from less energetic merger events (smaller masses or minor mergers in massive systems) or in the case of higher-redshift clusters, where inverse Compton (IC) losses become severe compared to synchrotron losses (Cassano et al. 2006; Brunetti et al. 2008). Radio halos in the very early or very late stages of their lifetime are also expected to be underluminous and have particularly steep spectra (Donnert et al. 2013). Regarding A1451 and Z0634, these scenarios are disfavoured by the evidence that they do not host radio halos with very steep spectra (Sect. 5.1).

For the two underluminous radio halos presented in this paper we propose two possibilities. They could be small radio halos, originating from minor mergers where the bulk of the turbulence is generated and dissipated in small volumes. The fact that the X-ray morphology of these two clusters is not extremely disturbed may suggest that they are undergoing minor mergers. In this case, the bulk of the turbulence may be generated and dissipated in small volumes resulting in relatively flat radio spectra, despite the relatively low amount of energy involved in the merger events. Alternatively, they could be "off-state" halos where the emission is primarily maintained by the continuous injection of secondary electrons of hadronic interactions in the ICM. Possible detections of "off-state" radio halos have been reported in the literature (e.g. Brown et al. 2011; Bonafede et al. 2014). The relatively flat spectra and the small sizes of these two newly discovered radio halos would be in line with both these scenarios.

\subsection{The candidate radio relic}

The elongated and arc-like morphology of the source detected North of A1451 (Fig. 4), together with its spectral index $(\alpha \approx$ -1.1 , see Sect. 3.2) suggest that it could be a radio relic. With a radio power of $P_{1.4 \mathrm{GHz}}=1.13 \pm 0.06 \times 10^{24} \mathrm{~W} / \mathrm{Hz}$ (Sect. 3.2), this source would follow the radio-luminosity-mass correlation found by de Gasperin et al. (2014) for radio relics. However, if this source is at the cluster redshift, its projected distance from the cluster centre is $\sim 3 \mathrm{Mpc}$, which would make it the most distant relic from the cluster centre ever detected (Vazza et al. 2012; de Gasperin et al. 2014). The virial radius of A1451 is $R_{v} \sim 2.5 \mathrm{Mpc}^{1}$, moreover, weak lensing studies show that its mass distribution is elongated in the N-S direction (see Fig. 3 in Cypriano et al. 2004), thus supporting the idea that we would be observing a radio relic located in the cluster's most external regions. The candidate radio relic in A1451 might be associated to an external shock, rather than a merger shock. Accretion/external shocks are the result of the continuous accretion

\footnotetext{
1 To derive the virial radius, $R_{v}$, we converted $M_{500}$ to $M_{\mathrm{vir}}$, by assuming an NFW profile (e.g. Navarro et al. 1997) for the dark matter halos and the concentration-mass relation in Duffy et al. (2008).
} 

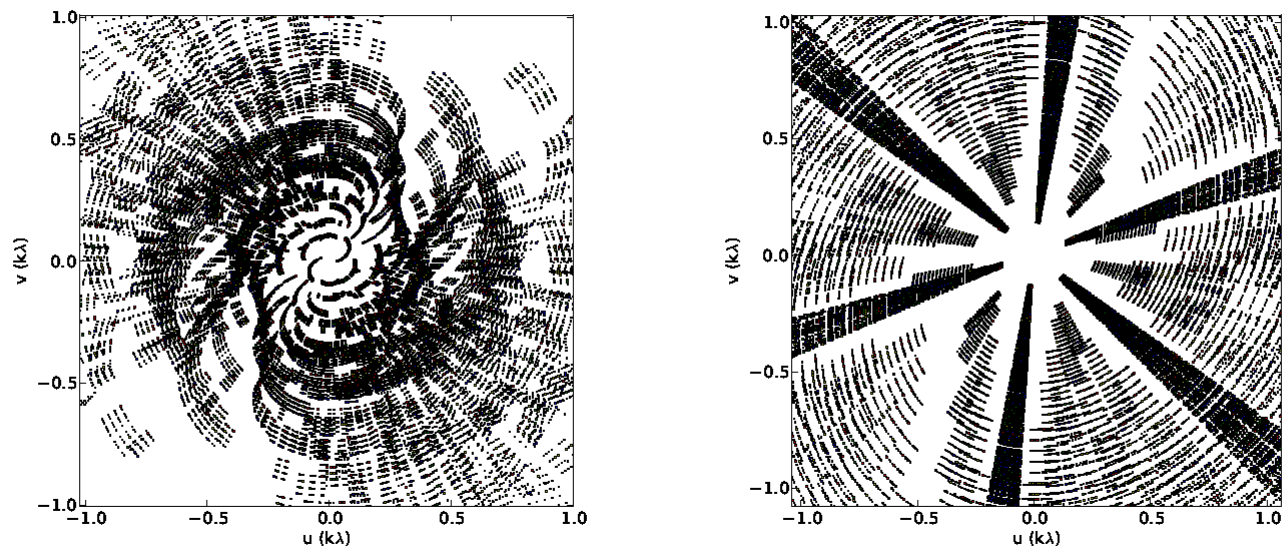

Fig. 10. Inner $u v$-coverage of the GMRT $320 \mathrm{MHz}$ observation (left) and of the JVLA D array observation (right) of Z0634.

of matter at several Mpc distance from the cluster centre. They are typically much stronger than merger shocks, since they develop in regions where the medium is much cooler than in clusters. On the other hand, they propagate through regions with extremely low gas densities. The energy flux dissipated at these shocks (both into heating of the ICM and CR acceleration) is much smaller than that of merger shocks that are generally faster and propagate into denser ICM (Ryu et al. 2003; Vazza et al. 2009). This makes external shocks very difficult to detect, unless they encounter and re-accelerate some clouds of relativistic plasma during their propagation. For instance, the candidate relic in A1451 could be instigated by an external shock that propagates through the lobes of a dead radio galaxy (Enßlin \& Gopal-Krishna 2001). Source S (Fig. 4) might be responsible for the presence of such ghost plasma. Unfortunately no spectroscopic redshift is available for this source. The 2 MASS catalogue (Skrutskie et al. 2006) reports a $K_{\mathrm{s}}$ magnitude of 15.03. Using the $K_{\mathrm{s}}-z$ relation by Willott et al. (2003) we estimated that the redshift of source $S$ is $z=0.3 \pm 0.1$, which does not exclude the possibility that it is close to the cluster redshift. We note that this scenario is in agreement with the fact that the candidate relic is well below the LLS-cluster-centre distance correlation by de Gasperin et al. (2014). Such a correlation has been found for double radio relics, associated to merger-driven shocks. In case of shocks crossing a pre-existing cloud of relativistic plasma, the size of the relic is determined by the size of that cloud which, in general, is expected to extend much less than the surface crossed by external shocks. Clearly, another possibility is that this source is a background giant radio galaxy, although the lack of a significant spectral gradient along the E-W direction disfavours this hypothesis.

Future studies on the polarisation properties of this object will be crucial to establish its nature.

\subsection{Head tail}

The HT radio galaxy in Z0634 shows very interesting properties. The spectral index of the tail between 320 and $1500 \mathrm{MHz}$ shows a continuous steepening from $\sim-0.65$ to $\sim-2.4$. In order to understand if such a steepening is consistent with pure radiative ageing of the electrons, we attempt to extract both the spectral index and the surface brightness with distance. We selected six circular regions along the tail and a final rectangular one (Fig. 7). The size of the circular regions is $\sim 1.5$ times the beam area. We selected a larger rectangular area on the last portion of the tail that is only marginally visible at $1.5 \mathrm{GHz}$ in order to have a better
$\mathrm{S} / \mathrm{N}$. We did not consider the very last part of the tail (West of C in Fig. 7, left) because it is only visible at $320 \mathrm{MHz}$ with a poor $\mathrm{S} / \mathrm{N}$.

The radio surface brightness and the spectral index show a peculiar evolution along the tail (Fig. 12). In the first part of the tail $(\mathrm{A} \rightarrow \mathrm{B})$ they progressively decline as expected due to radiative losses of a population of relativistic electrons that have been accelerated in the head of the radio galaxy (in A). In the presence of synchrotron and IC losses only, the (emitted) break frequency is:

$$
\begin{aligned}
\nu_{\mathrm{b}}(\mathrm{GHz}) & =2.6 \frac{\tau(\mathrm{Gyr})^{-2} B(\mu \mathrm{G})}{\left(B(\mu \mathrm{G})^{2}+B_{\mathrm{CMB}}(\mu \mathrm{G})^{2}\right)^{2}} \\
& \propto \frac{\left(\eta \sin \theta_{\mathrm{O}} \sigma_{v}\right)^{2}}{B_{\mathrm{CMB}}^{3}} \frac{\psi}{\left(\frac{\psi^{2}}{3}+1\right)^{2}} d_{\perp}^{-2},
\end{aligned}
$$

where $\tau$ is the radiative age of the electrons, $B_{\mathrm{CMB}}$ is the equivalent magnetic field of the cosmic microwave background (CMB), $\sigma_{v}$ is the velocity dispersion of the cluster $\left(\sigma_{v} \sim 1400 \mathrm{~km} \mathrm{~s}^{-1}\right)$ and $\eta \sin \theta_{\mathrm{O}} \sigma_{v}$ is the (projected) velocity of the head. We note that $\eta \sin \theta_{\mathrm{O}}$ combines two unknowns: the velocity of the HT and the inclination of its orbit. In Eq. (3), we introduced the quantity $B=\psi B_{\mathrm{CMB}} / \sqrt{3}$, where $\psi$ parametrises the magnetic field of the tail. For $\psi=1$, the electrons emitting at the observing frequencies in a magnetic field $B$ are in the situation of minimum radiative losses and consequently maximum lifetime. In the simplest interpretation, the fact that the break frequency scales with $d^{-2}$ between A and B (Fig. 11) suggests that adiabatic losses and strong variations of $B$ do not play an important role at this stage, otherwise a faster increase of $v_{1} / v_{\mathrm{b}}$ would be observed. More quantitatively, assuming $\psi=1$ and $\eta \sin \theta_{0}=1$, in Fig. 12 we show the measured spectral index and brightness as a function of projected distance. Also in this case, a simple scenario of pure radiative losses in the region $\mathrm{A}-\mathrm{B}$ can explain the data. We note that there is degeneracy in the parameters: from Eq. (3), one finds that the same match can be obtained with different choices of the parameters $\eta$ and $\psi$, that is, $B$, and $\theta_{\mathrm{O}}$ under the condition $\eta^{2} \sin ^{2} \theta_{\mathrm{O}} \frac{\psi}{\left(\psi^{2} / 3+1\right)^{2}}=9 / 16$.

In B (Fig. 7), the surface brightness is boosted up by a factor of 3.5-4, while the spectral index stops declining and remains almost constant for $\sim 100 \mathrm{kpc}$, corresponding to $\sim 60 \mathrm{Myr}$ (if we consider $\left.\eta \sin \theta_{\mathrm{O}}=1\right)^{2}$. Then, from B to the end of the tail (C),

\footnotetext{
2 We point out that these values are only indicative, since they depend on the sampling adopted in Fig. 7.
} 


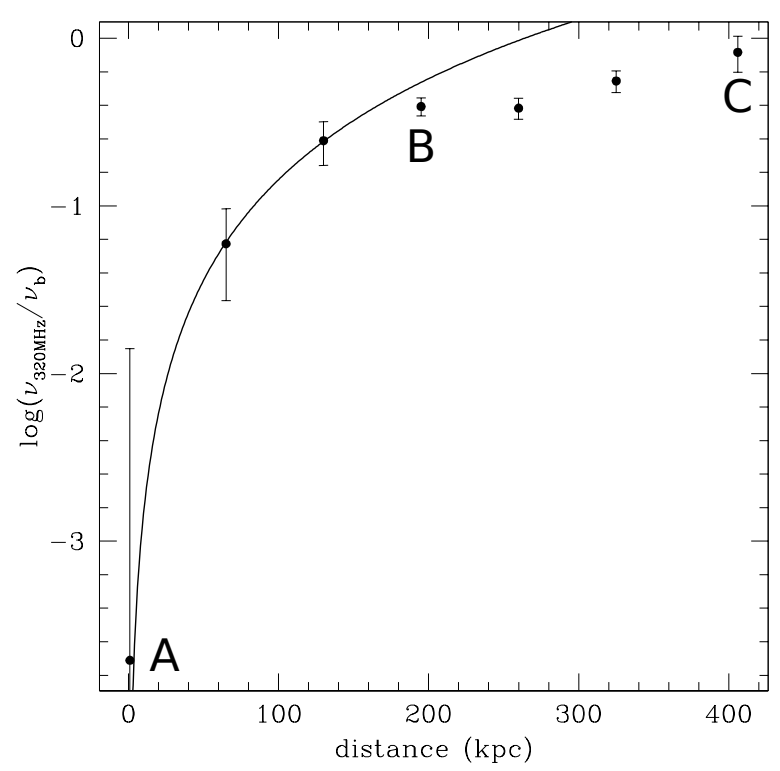

Fig. 11. $v_{320 \mathrm{MHz}} / v_{\mathrm{b}}$ vs. distance from the "head" of the HT galaxy. The line represents $v_{\mathrm{b}} \propto d^{-2}$. For display purposes the curve is normalised to the third data point.

both the surface brightness and the spectral index of the tail begin again to decrease and steepen, respectively. The possibility that we are seeing two HT radio galaxies chasing each other is excluded by the B array image (Fig. 5, right panel), where the bright spot is resolved and appears as part of the diffuse emission of the tail. Moreover, the spectral index in B is very steep and inconsistent with that of synchrotron radiation from a young plasma. On the other hand, the properties of the tail suggest that around region $\mathrm{B}$, the electrons in the tail are currently experiencing re-energisation and possibly that the magnetic field in the tail is also amplified.

The most natural mechanism that may explain these properties in the ICM is the interaction between a shock and the radio tail (e.g. Pfrommer \& Jones 2011). A schematic view is illustrated in Fig. 13. The HT galaxy is travelling outward from the cluster centre with an angle $\theta_{\mathrm{O}}$ to the line of sight. A shock front impacts the tail first and gets to the point B where we are currently observing it. When the shock passes through the tail the adiabatic compression re-energises a population of aged electrons and increases the magnetic field. This re-energisation leads to an increase of the synchrotron brightness in $\mathrm{B}$ and affects the observed spectral properties. In the downstream region $(B \rightarrow C)$ the electrons have begun to cool down again in the new magnetic field, but the ageing time has to be computed from the moment of the shock passage and it no longer depends on the velocity of the tail. Of course, the dynamics of the HT-shock interaction can be very complicated and would require ad hoc simulations that are beyond the scope of this work. For example, the shock crossing may change the inclination of the shocked portion of the tail, implying that projection effect may play an important role. In addition, after the shock passage, the tail may experience some expansion together with the gas downstream, and adiabatic expansion may induce a strong spectral steepening in the tail at later stages due to adiabatic losses and the decline of the magnetic field.

The system is complicated and unfortunately our observations cannot constrain all model parameters. Here we limit ourselves to demonstrate that, in the case of a weak shock travelling at a large angle with respect to the plane of the sky, this scenario has the potential to explain the observed properties of the tail (see Appendix for the formalism). To do that, we assume the simplest case where the shock is moving along the line-ofsight and where $\psi(t=0)=1$ (i.e. electrons upstream in the tail have minimum energy losses), $\eta \sin \theta_{\mathrm{O}}=1$, and the velocity dispersion of the cluster $\sigma_{v}=1400 \mathrm{~km} \mathrm{~s}^{-1}$. We also assume that particles are continuously injected into the core region of the $\mathrm{HT}$ at a rate that is constant with time (Appendix). Figure 12 (left panel) shows that this situation matches the observed spectrum upstream $(\mathrm{A} \rightarrow \mathrm{B})$, with the degeneracy of the parameters upstream given by Eq. (3). The evolution of the magnetic filed in the downstream region is given by Eq. (A.5), considering $B(t)=B\left(t_{0}\right) C_{t}^{2 / 3}$, and the conversion from time to projected distance is given by Eq. (A.7). The evolution of particles downstream subject to adiabatic compression and (time-variable) radiative losses is calculated in the Appendix. In Fig. 12 we show the model results assuming $\mathcal{M}=1.7$ and $\theta_{\mathrm{O}}=60^{\circ}$, thus the velocity of the tail is $v=\sigma_{v} / \sin 60^{\circ} \approx 1600 \mathrm{~km} \mathrm{~s}^{-13}$. The two curves assume $\Gamma=4 / 3$ (relativistic plasma) and $\Gamma=5 / 3$ (the case where most of the energy of the plasma in the tail is contributed by thermal gas). Given the number of model parameters (Appendix) and the uncertainties on the dynamics of the interaction between the tail and the shock, a full extrapolation of the model parameters is not meaningful at this stage. However, from Fig. 12, we can conclude that the proposed scenario can naturally explain the observations for reasonable choices of model parameters (i.e. for a velocity of the tail that is of the order of the cluster velocity dispersion), assuming a weak shock with Mach number $<2$, similar to most shocks detected in galaxy clusters. In this respect, a simple estimate of the maximum Mach number can be obtained neglecting losses during the shock compression phase (e.g. Markevitch et al. 2005, see also Appendix). For $\Gamma=5 / 3$ (for $\Gamma=4 / 3$ the limit is more stringent) and for a shock travelling at a large angle to the plane of the sky this is:

$\mathcal{M} \leq\left(\frac{3}{4\left(\frac{I_{\mathrm{u}}}{I_{\mathrm{d}}}\right)^{\frac{3}{2 \delta}}-1}\right)^{\frac{1}{2}}$,

which gives $\mathcal{M} \leq 1.58$ by assuming the observed brightness jump $I_{\mathrm{d}} / I_{\mathrm{u}} \sim 4$ and a spectrum of the emitting electrons $\delta=3.5$; the allowed Mach number is slightly larger when radiative losses during compression are properly included (see Fig. 12) ${ }^{4}$.

We also note that no shock is clearly visible at the position $B$ in the currently available X-ray image, suggesting that if a shock is present it should be weak and/or moving in a direction that is significantly inclined with respect to the plane of the sky.

\section{Conclusions}

In this paper we present deep GMRT $320 \mathrm{MHz}$ observations and JVLA $1.5 \mathrm{GHz}$ observations in B and D array of the galaxy clusters A1451 and Z0634. We summarise our results below:

- We discovered a radio halo at the centre of A1451. The radio halo has a LLS of $\sim 750 \mathrm{kpc}\left(r_{\mathrm{e}} \sim 140 \mathrm{kpc}\right.$ based on the injection of fake radio halos, Sect. 5.1) and a $k$-corrected radio

3 We note that for a given magnetic field $\psi$ the normalisation of the synchrotron brightness depends on the normalisation of the electron spectrum, $K_{\mathrm{e}}$ (Appendix), that is a free parameter.

4 We point out that given the observed spectrum and the Mach numbers used in our calculations, even in the case $\Gamma=5 / 3$ (in this case particles inside the HT will be shocked) the process of shock acceleration is subdominant with respect to compression. 

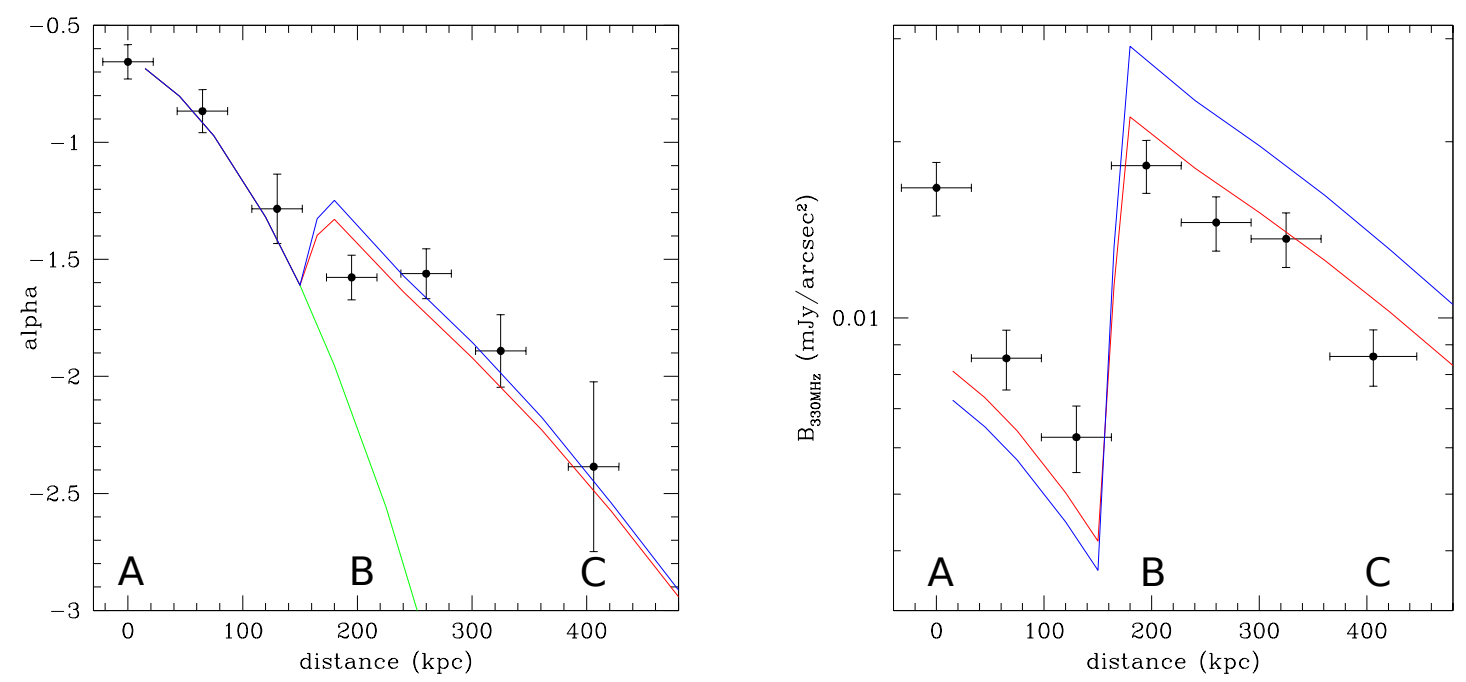

Fig. 12. Left: spectral index of the HT vs. distance from the "head". The green line is the classical model of synchrotron ageing of a population of electrons that have been accelerated in the "head". The injection spectrum is assumed to match the spectrum in the head of the tail. Right: radio surface brightness vs. distance; model normalisation ( $K_{\mathrm{e}}$ in Appendix) is chosen to best illustrate the comparison with the data. A, B and C refer to Fig. 8. In both panels the model parameters are: $\mathcal{M}=1.7, \theta_{\mathrm{SO}}=\pi / 2, a=1, c_{\mathrm{S}}=1330 \mathrm{~km} \mathrm{~s}^{-1}, \sigma_{v}=1400 \mathrm{~km} \mathrm{~s}^{-1}, \psi(t=0)=1, \eta \sin \theta_{\mathrm{O}}=1$, with $\theta_{\mathrm{O}}=60^{\circ}$ and $\theta_{\mathrm{SO}}=90^{\circ}, \Gamma=5 / 3$ (red line) and $\Gamma=4 / 3$ (blue line).

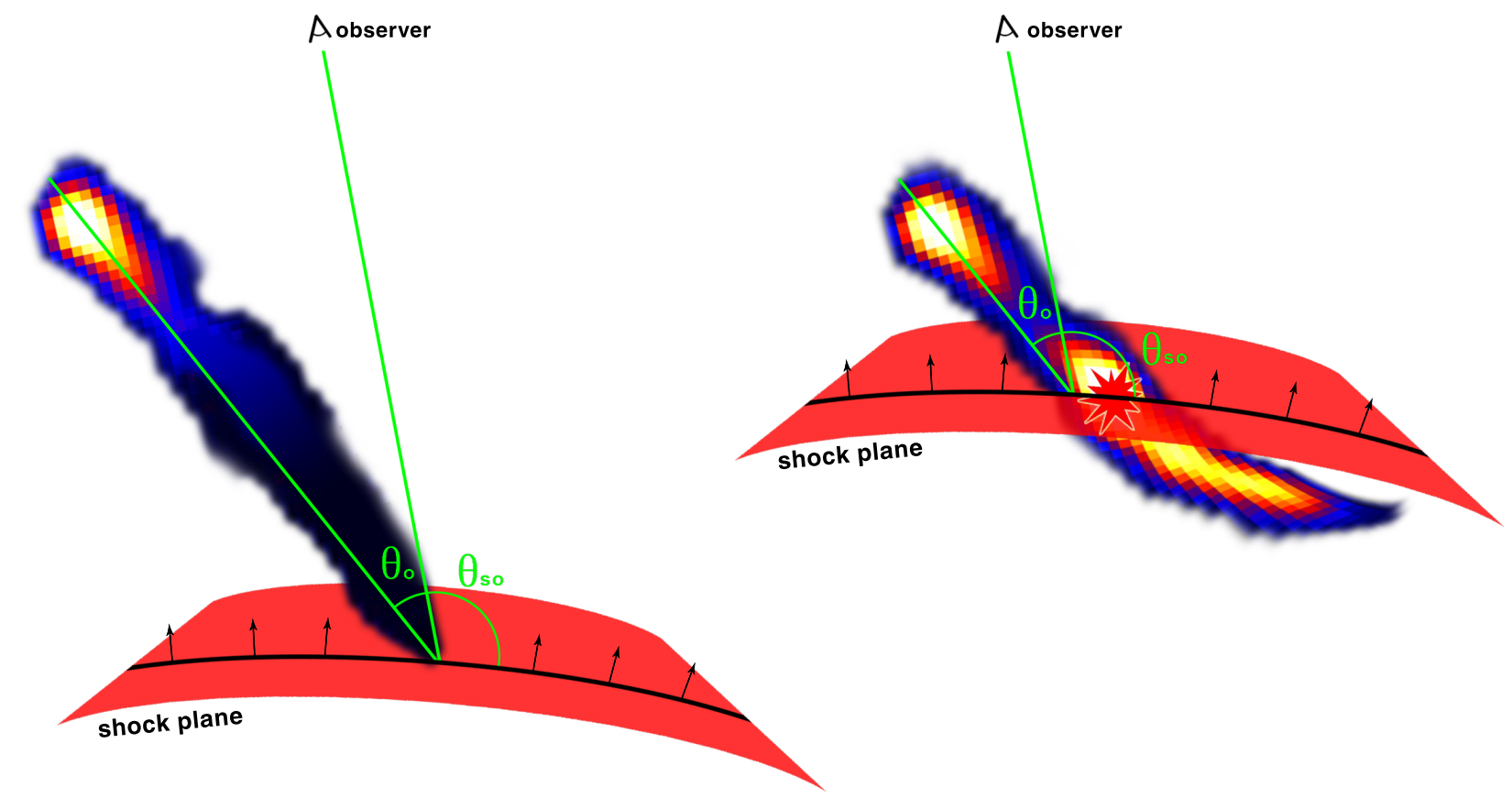

Fig. 13. Cartoon of the interaction between the shock front and the HT. Before the interaction (left) the surface brightness of the HT progressively declines from the head to the tail. The right panel represents the current situation: the shock has passed through the last part of the tail that appears brighter because of the re-energisation undergone by the electrons. The bright spot is where the tail is currently shocked. The shock passage also caused the bending of the tail in the direction parallel to the shock itself.

power at $1.4 \mathrm{GHz} P_{1.4 \mathrm{GHz}}=6.4 \pm 0.7 \times 10^{23} \mathrm{~W} / \mathrm{Hz}$. We estimated that the spectral index of this radio halo between 320 and $1500 \mathrm{MHz}$ is in the range $-1.3<\alpha<-1.1$.

- We discovered that Z0634 hosts a radio halo. It has a LLS of $\sim 600 \mathrm{kpc}\left(r_{\mathrm{e}} \sim 130 \mathrm{kpc}\right.$ based on the injection of fake radio halos, Sect. 5.1) and a $k$-corrected radio power at $1.4 \mathrm{GHz}$ $P_{1.4 \mathrm{GHz}}=3.1 \pm 0.2 \times 10^{23} \mathrm{~W} / \mathrm{Hz}$. Its spectral index between 320 and $1500 \mathrm{MHz}$ is $-1.3<\alpha<-0.95$.

- Both the radio halos in A1451 and Z0634 are underluminous with respect to the radio power-mass correlation found by Cassano et al. (2013) and Martinez Aviles et al. (2016) (Fig. 9), similarly to a few other clusters reported in the literature (Venturi et al. 2007, 2013; Sommer et al. 2017; Bonafede et al. 2015). That region of the $P_{1.4 \mathrm{GHz}}-M_{500} \mathrm{di}-$ agram is usually occupied either by upper limits or by ultra steep spectrum radio halos, however, A1451 and Z0634 do not seem to host radio halos with steep spectra (Sect. 5.1). In the framework of current theoretical models, they could be small radio halos generated during minor mergers where turbulence has been dissipated in smaller volumes, or "offstate" radio halos originating from hadronic collisions in the ICM.

- We detected an elongated diffuse source $\sim 15$ arcmin North of the centre of A1451 that we classified as a candidate 
radio relic. This source has a LAS of $\sim 6.5$ arcmin and a $k$ corrected radio power at $1.4 \mathrm{GHz} P_{1.4 \mathrm{GHz}}=1.13 \pm 0.06 \times$ $10^{24} \mathrm{~W} / \mathrm{Hz}$. Its spectral index between 320 and $1500 \mathrm{MHz}$ is $\alpha=-1.1 \pm 0.1$. If this source is at the cluster redshift, its projected distance from the cluster centre is $\sim 3 \mathrm{Mpc}$, which would be the largest distance ever measured for a relic (e.g. Vazza et al. 2012; de Gasperin et al. 2014). We propose that this relic could be the result of an accretion/external shock propagating through some clouds of ghost plasma. The fact that this source is well below the LLS-cluster centre distance correlation by de Gasperin et al. (2014) is in agreement with the accretion shock scenario. Polarisation information will be fundamental to address the nature of this object.

- Z0634 hosts a HT radio galaxy with intriguing characteristics. The spectral index between 320 and $1500 \mathrm{MHz}$ steepens from the head to the tail (from $\alpha=-0.65$ to $\alpha=-2.4$ ), however, this steepening is not consistent with a purely radiative ageing of electrons. Moreover, there is a spot of high surface brightness along the tail and the second part of the tail, after the spot, is brighter than the first part, before the spot. We have shown that the interaction of the HT with a weak shock $(\mathcal{M} \lesssim 2)$ moving toward the outskirts of the cluster can explain the observed properties.

Acknowledgements. The authors thank the referee for useful comments, which have improved the presentation of the paper. V.C. thanks G. Conte who produced the cartoon in Fig. 13. V.C., G.B., R.C., T.V. acknowledge partial support from grant PRIN INAF 2014. R.K. acknowledges support from the DST-INSPIRE Faculty award by the Department of Science and Technology, Government of India. The National Radio Astronomy Observatory is a facility of the National Science Foundation operated under cooperative agreement by Associated Universities, Inc. We thank the staff of the GMRT, who made these observations possible. GMRT is run by the National Centre for Radio Astrophysics of the Tata Institute of Fundamental Research. The scientific results reported in this article are based in part on data obtained from the Chandra Data Archive. This research has made use of the NASA/IPAC Extragalactic Database (NED) which is operated by the Jet Propulsion Laboratory, California Institute of Technology, under contract with the National Aeronautics and Space Administration.

\section{References}

Ackermann, M., Ajello, M., Allafort, A., et al. 2010, ApJ, 717, L71 Ackermann, M., Ajello, M., Albert, A., et al. 2014, ApJ, 787, 18 Ackermann, M., Ajello, M., Albert, A., et al. 2016, ApJ, 819, 149 Bacchi, M., Feretti, L., Giovannini, G., \& Govoni, F. 2003, A\&A, 400, 465 Basu, K. 2012, MNRAS, 421, L112

Begelman, M. C., Rees, M. J., \& Blandford, R. D. 1979, Nature, 279, 770 Blasi, P., \& Colafrancesco, S. 1999, Astropart. Phys., 12, 169 Bonafede, A., Intema, H. T., Brüggen, M., et al. 2014, ApJ, 785, 1 Bonafede, A., Intema, H., Brüggen, M., et al. 2015, MNRAS, 454, 3391 Botteon, A., Gastaldello, F., Brunetti, G., \& Dallacasa, D. 2016, MNRAS, 460, L84

Brown, S., Emerick, A., Rudnick, L., \& Brunetti, G. 2011, ApJ, 740, L28 Brüggen, M., Bykov, A., Ryu, D., \& Röttgering, H. 2012, Space Sci. Rev., 166, 187

Brunetti, G., \& Blasi, P. 2005, MNRAS, 363, 1173

Brunetti, G., \& Jones, T. W. 2014, Int. J. Mod. Phys. D, 23, 1430007

Brunetti, G., \& Lazarian, A. 2011, MNRAS, 410, 127

Brunetti, G., Setti, G., Feretti, L., \& Giovannini, G. 2001, MNRAS, 320, 365

Brunetti, G., Venturi, T., Dallacasa, D., et al. 2007, ApJ, 670, L5

Brunetti, G., Giacintucci, S., Cassano, R., et al. 2008, Nature, 455, 944

Brunetti, G., Cassano, R., Dolag, K., \& Setti, G. 2009, A\&A, 507, 661

Brunetti, G., Blasi, P., Reimer, O., et al. 2012, MNRAS, 426, 956

Brunetti, G., Zimmer, S., \& Zandanel, F. 2017, MNRAS, 472, 1506

Cassano, R., Brunetti, G., \& Setti, G. 2006, MNRAS, 369, 1577

Cassano, R., Brunetti, G., Setti, G., Govoni, F., \& Dolag, K. 2007, MNRAS, 378, 1565

Cassano, R., Ettori, S., Giacintucci, S., et al. 2010, ApJ, 721, L82

Cassano, R., Brunetti, G., Norris, R. P., et al. 2012, A\&A, 548, A100

Cassano, R., Ettori, S., Brunetti, G., et al. 2013, ApJ, 777, 141

Cassano, R., Brunetti, G., Giocoli, C., \& Ettori, S. 2016, A\&A, 593, A81

Chandra, P., Ray, A., \& Bhatnagar, S. 2004, ApJ, 612, 974

Condon, J. J., Cotton, W. D., Greisen, E. W., et al. 1998, AJ, 115, 1693
Cuciti, V., Cassano, R., Brunetti, G., et al. 2015, A\&A, 580, A97

Cypriano, E. S., Sodré, Jr., L., Kneib, J.-P., \& Campusano, L. E. 2004, ApJ, 613, 95

de Gasperin, F., van Weeren, R. J., Brüggen, M., et al. 2014, MNRAS, 444, 3130 Dennison, B. 1980, ApJ, 239, L93

Donnert, J., Dolag, K., Brunetti, G., \& Cassano, R. 2013, MNRAS, 429, 3564 Eckert, D., Jauzac, M., Vazza, F., et al. 2016, MNRAS, 461, 1302

Eilek, J. A., Burns, J. O., O’Dea, C. P., \& Owen, F. N. 1984, ApJ, 278, 37 Ensslin, T. A., Biermann, P. L., Klein, U., \& Kohle, S. 1998, A\&A, 332, 395 Enßlin, T. A., \& Gopal-Krishna 2001, A\&A, 366, 26

Feretti, L., \& Venturi, T. 2002, in Merging Processes in Galaxy Clusters, eds. L. Feretti, I. M. Gioia, \& G. Giovannini, Astrophys. Space Sci. Libr., 272, 163

Feretti, L., Fusco-Femiano, R., Giovannini, G., \& Govoni, F. 2001, A\&A, 373, 106

Feretti, L., Giovannini, G., Govoni, F., \& Murgia, M. 2012, A\&ARv, 20, 54

Giacintucci, S., \& Venturi, T. 2009, A\&A, 505, 55

Hill, J. M., \& Longair, M. S. 1971, MNRAS, 154, 125

Jaffe, W. J., \& Perola, G. C. 1973, A\&A, 26, 423

Jeltema, T. E., \& Profumo, S. 2011, ApJ, 728, 53

Kaiser, C. R., Dennett-Thorpe, J., \& Alexander, P. 1997, MNRAS, 292, 723

Kale, R., Venturi, T., Giacintucci, S., et al. 2013, A\&A, 557, A99

Kale, R., Venturi, T., Giacintucci, S., et al. 2015, A\&A, 579, A92

Kang, H., Ryu, D., \& Jones, T. W. 2012, ApJ, 756, 97

Lane, W. M., Kassim, N. E., Ensslin, T. A., Harris, D. E., \& Perley, R. A. 2002, AJ, 123, 2985

Liang, H., Hunstead, R. W., Birkinshaw, M., \& Andreani, P. 2000, ApJ, 544, 686

Mao, M. Y., Johnston-Hollitt, M., Stevens, J. B., \& Wotherspoon, S. J. 2009, MNRAS, 392, 1070

Markevitch, M., Govoni, F., Brunetti, G., \& Jerius, D. 2005, ApJ, 627, 733

Martinez Aviles, G., Ferrari, C., Johnston-Hollitt, M., et al. 2016, A\&A, 595, A116

McMullin, J. P., Waters, B., Schiebel, D., Young, W., \& Golap, K. 2007, in Astronomical Data Analysis Software and Systems XVI, eds. R. A. Shaw, F. Hill, \& D. J. Bell, ASP Conf. Ser., 376, 127

Miley, G. 1980, ARA\&A, 18, 165

Mohan, N., \& Rafferty, D. 2015, Astrophysics Source Code Library [record ascl: 1502.007]

Motl, P. M., Hallman, E. J., Burns, J. O., \& Norman, M. L. 2005, ApJ, 623, L63 Murgia, M., Govoni, F., Markevitch, M., et al. 2009, A\&A, 499, 679

Nagai, D. 2006, ApJ, 650, 538

O’Dea, C. P., \& Owen, F. N. 1986, ApJ, 301, 841

Orrú, E., Murgia, M., Feretti, L., et al. 2007, A\&A, 467, 943

Owen, F. N., \& Rudnick, L. 1976, ApJ, 205, L1

Owen, F. N., Rudnick, L., Eilek, J., et al. 2014, ApJ, 794, 24

Perley, R. A., \& Butler, B. J. 2013, ApJS, 204, 19

Petrosian, V. 2001, ApJ, 557, 560

Pfrommer, C., \& Jones, T. W. 2011, ApJ, 730, 22

Piffaretti, R., Arnaud, M., Pratt, G. W., Pointecouteau, E., \& Melin, J.-B. 2011, A\&A, 534, A109

Pinkney, J., Burns, J. O., \& Hill, J. M. 1994, AJ, 108, 2031

Pinzke, A., Oh, S. P., \& Pfrommer, C. 2013, MNRAS, 435, 1061

Pinzke, A., Oh, S. P., \& Pfrommer, C. 2017, MNRAS, 465, 4800

Planck Collaboration XXIX. 2014, A\&A, 571, A29

Roettiger, K., Burns, J. O., \& Stone, J. M. 1999, ApJ, 518, 603

Rudnick, L., \& Lemmerman, J. A. 2009, ApJ, 697, 1341

Russell, H. R., van Weeren, R. J., Edge, A. C., et al. 2011, MNRAS, 417, L1

Ryu, D., Kang, H., Hallman, E., \& Jones, T. W. 2003, ApJ, 593, 599

Scaife, A. M. M., \& Heald, G. H. 2012, MNRAS, 423, L30

Shimwell, T. W., Markevitch, M., Brown, S., et al. 2015, MNRAS, 449, 1486

Skrutskie, M. F., Cutri, R. M., Stiening, R., et al. 2006, AJ, 131, 1163

Sommer, M. W., Basu, K., Intema, H., et al. 2017, MNRAS, 466, 996

Valtchanov, I., Murphy, T., Pierre, M., Hunstead, R., \& Lémonon, L. 2002, A\&A, 392, 795

van Weeren, R. J., Brunetti, G., Brüggen, M., et al. 2016, ApJ, 818, 204

van Weeren, R. J., Andrade-Santos, F., Dawson, W. A., et al. 2017, Nat. Astron., 1,0005

Vazza, F., \& Brüggen, M. 2014, MNRAS, 437, 2291

Vazza, F., Brunetti, G., \& Gheller, C. 2009, MNRAS, 395, 1333

Vazza, F., Brüggen, M., van Weeren, R., et al. 2012, MNRAS, 421, 1868

Venturi, T., Giacintucci, S., Brunetti, G., et al. 2007, A\&A, 463, 937

Venturi, T., Giacintucci, S., Dallacasa, D., et al. 2008, A\&A, 484, 327

Venturi, T., Giacintucci, S., Dallacasa, D., et al. 2013, A\&A, 551, A24

Wellington, K. J., Miley, G. K., \& van der Laan, H. 1973, Nature, 244, 502

Willott, C. J., Rawlings, S., Jarvis, M. J., \& Blundell, K. M. 2003, MNRAS, 339, 173

Willson, M. A. G. 1970, MNRAS, 151, 1

Zandanel, F., \& Ando, S. 2014, MNRAS, 440, 663 


\section{Appendix A}

The evolution of the electron spectrum in the tail assuming radiative losses and compression (expansion) is (Kaiser et al. 1997; Enßlin \& Gopal-Krishna 2001):

$$
\frac{\partial N}{\partial t}=\frac{\partial}{\partial \gamma}\left\{N\left[\frac{4 \sigma_{T}}{3 m_{\mathrm{e}} c}\left(\frac{B^{2}}{8 \pi}+\frac{B_{\mathrm{CMB}}^{2}}{8 \pi}\right) \gamma^{2}+\frac{1}{3} \frac{\dot{V}}{V} \gamma\right]\right\},
$$

where the first term accounts for radiative (synchrotron and IC) losses and the second term for adiabatic compression or expansion.

If electrons have an initial energy distribution in the form $N(\gamma, 0)=k_{\mathrm{e}} \gamma^{-\delta}$ (with $\gamma$ between $\gamma_{\min }$ and $\gamma_{\max }$ such that $\gamma_{\min } \ll$ $\gamma_{r} \ll \gamma_{\max }, \gamma_{r}$ being the typical energy of the electrons emitting at 330 and $1400 \mathrm{MHz}$ ) and if we define the compression factor with time $C_{t}=V_{0} / V(t)$, the magnetic field will evolve with time according to $B(t)=B(0) C_{t}^{2 a / 3}$, where $a=1$ in the case of isotropic compression/expansion and the solution of Eq. (A.1) is :

$N(\gamma, t)=K_{\mathrm{e}} C_{t}^{\frac{\delta+2}{3}} \gamma^{-\delta}\left(1-\frac{\gamma}{\gamma_{* t}}\right)^{\delta-2}$

where

$\gamma_{* t}^{-1}=\frac{\sigma_{t} B_{\mathrm{CMB}}^{2}}{6 \pi m_{\mathrm{e}} c} \int_{0}^{t} \mathrm{~d} x\left(1+\frac{\psi_{x}^{2}}{3}\right)\left(\frac{C_{x}}{C_{t}}\right)^{\frac{1}{3}}$

where we have introduced the quantity

$\psi_{t}=\frac{B_{0}}{B_{\mathrm{CMB}} / \sqrt{3}} C_{t}^{\frac{2 a}{3}}$,

where $\psi_{t}=1$ is the case where radiative losses of electrons emitting at a given frequency in a given magnetic field are minimised.

Following Enßlin \& Gopal-Krishna (2001), we assume three phases: (i) an initial phase where electrons simply age $\left(t \leq t_{1}\right)$; (ii) a phase where the shock compresses the tail $\left(t_{1} \leq t \leq t_{2}\right.$, where $t_{2}-t_{1}=\Phi /\left(\mathcal{M} c_{\mathrm{s}}\right), \Phi$ is the thickness of the tail, $c_{\mathrm{s}}$ is the sound speed and $\mathcal{M}$ is the Mach number); and (iii) a new phase of cooling of the electrons after shock passage $\left(t>t_{2}\right)$. Under this assumptions the compression factor is

$$
\begin{cases}C_{t}=1 & t \leq t_{1} \\ C_{t}=1+\left(C_{t_{2}}-1\right) \frac{t-t_{1}}{t_{2}-t_{1}} & t_{1}<t \leq t_{2} \\ C_{t}=C_{t_{2}}=\frac{(\Gamma+1) \mathcal{M}^{2}}{(\Gamma-1) \mathcal{M}^{2}+2} & t>t_{2} .\end{cases}
$$

$\Gamma$ is the adiabatic index of the gas into the tail. The resulting synchrotron emissivity is:

$$
J(v, t)=\frac{e^{3} \sqrt{3}}{m_{\mathrm{e}} c^{2}} \int_{0}^{\frac{\pi}{2}} \mathrm{~d} \theta\left(\sin ^{2} \theta\right) B \int \mathrm{d} \gamma N(\gamma, t) x \int_{x}^{\infty} K_{5 / 3}(z) \mathrm{d} z,
$$

where $x=\frac{4 \pi m_{\mathrm{e}} c v}{3 e B \sin \theta \gamma^{2}}$ and $K_{5 / 3}(z)$ is the $5 / 3$ modified Bessel function.

Given the geometry in Fig. 13, the conversion from time to projected distance is given by

$$
\begin{cases}d_{\perp}=\eta \sigma_{v} \sin \theta_{\mathrm{O}} t & t \leq t_{2} \\ d_{\perp}=c_{\mathrm{S}} \mathcal{M} \frac{\sin \theta_{\mathrm{O}}}{\cos \left(\theta_{\mathrm{SO}}+\theta_{\mathrm{O}}-\frac{\pi}{2}\right)}\left(t-t_{2}\right) & t>t_{2},\end{cases}
$$

where $\eta \sigma_{V}$ is the velocity of the head of the tail. The synchrotron brightness is:

$I\left(v, d_{\perp}\right)=\frac{1}{2 \pi} \int_{d_{\perp}} \frac{J(v, r(t)) r \mathrm{~d} r}{\sqrt{r^{2}-d_{\perp}^{2}}}$,

where the integral is done along the tail intercepted by the line of sight.

The model parameters are: the velocity of the HT $(\eta)$, the magnetic field $(\psi)$, the normalisation of the electron spectrum $\left(K_{\mathrm{e}}\right)$, the geometry of the problem $\left(\theta_{\mathrm{O}}, \theta_{\mathrm{SO}}\right)$ and the shock Mach number $(\mathcal{M})$. In comparing the model with the data, we focus on key observables: the spatial behaviour of the synchrotron spectral index and of the synchrotron brightness. A comparison with these properties is given in Fig. 12 for reference model parameters. In particular, a key observable that constrains the shock Mach number in the model is the observed jump of the synchrotron brightness across the shock, that is, by the ratio of the downstream and upstream brightness, $I_{\mathrm{d}} / I_{\mathrm{u}} ; I_{\mathrm{d}}$ and $I_{\mathrm{u}}$ are given by Eq. (A.8) evaluated at time $=t_{2}$ and $t_{1}$, respectively. A useful upper limit to the expected brightness jump can be easily derived by assuming that energy losses during the compression phase can be neglected. In this case, if we assume a power-law spectrum of electrons, $N(\gamma) \propto \gamma^{-\delta}$, the ratio $I_{\mathrm{d}} / I_{\mathrm{u}}=C_{t_{2}}^{2 \delta / 3+1} l_{\mathrm{d}} / l_{\mathrm{u}}$ (e.g. Markevitch et al. 2005) where $l_{\mathrm{d}}$ and $l_{\mathrm{u}}$ are the downstream and upstream thickness intercepted by the line of sight, respectively. For reference, this gives $I_{\mathrm{d}} / I_{\mathrm{u}}=C_{t_{2}}^{2 \delta / 3+1}$ and $=C_{t_{2}}^{2 \delta / 3}$ in the situation of shock travelling at small and large angles to the plane of the sky, respectively. 